\title{
Vibrational modes of hydraulic fractures: Inference of fracture geometry from resonant frequencies and attenuation
}

Bradley P. Lipovsky ${ }^{1}$ and Eric M. Dunham ${ }^{1,2}$

\author{
${ }^{1}$ Department Geophysics, Stanford \\ University, Stanford, CA, USA. \\ ${ }^{2}$ Institute for Computational and \\ Mathematical Engineering, Stanford \\ University, Stanford, CA, USA.
}


4 Abstract. Oscillatory seismic signals arising from resonant vibrations of ${ }_{5}$ hydraulic fractures are observed in many geologic systems, including volca-

noes, glaciers and ice sheets, and hydrocarbon and geothermal reservoirs. To better quantify the physical dimensions of fluid-filled cracks and properties of the fluids within them, we study wave motion along a thin hydraulic fracture waveguide. We present a linearized analysis, valid at wavelengths greater than the fracture aperture, that accounts for quasi-static elastic deformation of the fracture walls, as well as fluid viscosity, inertia, and compressibility. In the long-wavelength limit, anomalously dispersed guided waves known as crack or Krauklis waves propagate with restoring force from fracture wall elasticity. At shorter wavelengths, the waves become sound waves within the fluid channel. Wave attenuation in our model is due to fluid viscosity, rather than seismic radiation from crack tips or fracture wall roughness. We characterize viscous damping at both low frequencies, where the flow is always fully developed, and at high frequencies, where the flow has a nearly constant velocity profile away from viscous boundary layers near the fracture walls. Most observable seismic signals from resonating fractures likely arise in the boundary layer crack wave limit, where fluid-solid coupling is pronounced and attenuation is minimal. We present a method to estimate the aperture and length of a resonating hydraulic fracture using both the seismically observed quality factor and characteristic frequency. Finally, we develop scaling relations between seismic moment and characteristic frequency that might be useful when interpreting the statistics of hydraulic fracture events. 


\section{Introduction}

${ }_{27} \quad$ Fluids in Earth's subsurface are of great societal interest. Petroleum, fracturing, and

28

geothermal fluids are basic components of the energy system; magmatic fluids in volcanoes are associated with natural hazards; the fossilized remains of ancient volcanic intrusions provide insights into past tectonic environments; and liquid water in ice plays a critical role in the response of the cryosphere to a changing climate. Such fluids are commonly contained in fractures. Fractures are pervasive in geologic media and fluid-filled fractures are the dominant fluid pathway in media with low intrinsic permeability. In the cryosphere, fluid-filled fractures occur as glacial crevasses as well as thin sheets of water at the bed of glaciers [Creyts and Schoof, 2009]. In volcanoes, such fractures occur as magma-filled dikes and sills [Rubin, 1995], while in geothermal and hydrocarbon reservoirs they provide either pre-existing or stimulation-induced fracture space [Gale et al., 2007].

Because of the expense and sometimes impossibility of in situ measurements, the ability to characterize fluid properties and fracture geometry using the seismic wave field is thus highly desirable.

Seismic waves originating from fluid-filled fractures offer a window into these difficult to observe systems. Swarms of shallow $(<5 \mathrm{~km})$ volcanic long-period (LP; $0.2-2 \mathrm{~s})$ events represent a forecasting tool of the climactic stage of volcanic eruptions [Chouet et al., 1994; Chouet, 1996; Sparks et al., 2012]. Seismic signals in ice sheets have been used to infer the timing and propagation of a subglacial outburst flood beneath the West Antarctic Ice Sheet [Winberry et al., 2009]. Tary et al. [2014] relate seismograms recorded during reservoir stimulation to the geometry of induced hydraulic fractures in an unconventional 
$\mathrm{X}-4$ LIPOVSKY AND DUNHAM: RESONANCE OF HYDRAULIC FRACTURES

48

hydrocarbon reservoir. Although these geologic settings are diverse, observed seismic waveforms in each case share many common features.

The most notable attribute of seismic signals emanating from fluid-filled fractures is the presence of one or more characteristic frequencies. The Fourier spectra of such signals are therefore strongly peaked or band-limited. The characteristic frequencies sometimes, but not always, have a harmonic relationship. The presence of harmonic spectral peaks suggests resonance phenomena [Aki et al., 1977]. Observations typically have the lowest characteristic frequency $f_{1}$ in the range of 1 to $1000 \mathrm{~Hz}$ (Table 1 ). When interpreted as a fundamental resonant frequency, $f_{1}$ is expected to be related to a wave speed $c$ and fracture length $L$ by $f_{1} \sim c / L$. This relationship suggests a method for estimating the subsurface crack length $L$ using the seismically observable frequency $f_{1}$. The crux of such an analysis is the choice of the proper wave speed. Fluid-filled fractures act as dispersive wave guides, where waves experience dispersion due to the elasticity of the hydraulic fracture walls [Krauklis, 1962; Paillet and White, 1982; Chouet, 1986; Ferrazzini and Aki, 1987]. The frequency dependence of the wave speed must therefore be taken into account in order to correctly interpret observed resonant frequencies.

The speed of wave propagation is determined by the restoring forces acting along the fracture. There are two important end-member restoring force regimes (Figure 1a). In the limit that the fracture walls are rigid compared to a highly compressible fluid, disturbances are accommodated as sound waves with sound wave speed $c_{0} \equiv \sqrt{K_{f} / \rho_{0}}$ for fluid bulk modulus $K_{f}$ and nominal fluid density $\rho_{0}$. In the opposite limit, the crack walls are highly deformable and the fluid is nearly incompressible. Waves in this setting will propagate as 
crack (or Krauklis) waves with the dispersive crack wave speed [Krauklis, 1962],

$$
c_{w} \equiv\left(\frac{G^{*}}{\rho_{0}} w_{0} \omega\right)^{1 / 3} .
$$

Here $G^{*}=G /\left(1-\nu_{s}\right)$ for solid shear modulus $G$ and Poisson ratio $\nu_{s}, w_{0}$ is the unperturbed conduit half-width, and $\omega$ is angular frequency. Note that (1) provides sensitivity of $f_{1}$ to the aperture. However, even when fluid and solid material properties are known, observation of $f_{1}$ alone is insufficient to uniquely constrain both $L$ and $w_{0}$.

Seismic events due to fluid-filled fractures may have either an impulsive or a continuous nature. In volcano seismology, impulsive, monochromatic seismograms are termed long period or LP events; continuous, monochromatic seismograms are termed volcanic tremor. The latter may have a duration from minutes to days and sometimes months or longer [Chouet and Matoza, 2013]. Similar long duration, monochromatic seismic signals have been observed in Antarctic ice streams [Winberry et al., 2009] and during hydraulic fracturing of geothermal [Bame and Fehler, 1986; Ferrazzini et al., 1990] and tight gas reservoirs [Tary et al., 2014]. Events with an impulsive nature often show a gradual amplitude decay in the latter part of their wave train or coda.

Amplitude decay is described by a quality factor $Q$. The quality factor describes the damping of a resonating fracture and corresponds to the number of oscillations that an impulsive signal undergoes before decay to $e^{-\pi} \approx 4 \%$ of its original amplitude. Alternatively, sustained excitation in the source region may result in sustained oscillations. Such oscillations may not have a discernible decay time, and for such signals the quality factor may be measured from the width of a spectral peak. Observations generally constrain $Q$ in the range of 1 to 1000 (Table 1). The physical origin of amplitude decay lies in 
two mechanisms of attenuation: fluid viscosity and emitted seismic radiation $[A k i, 1984$;

Chouet, 1992].

The purpose of this work is to study the role of viscous damping of hydraulic fracture guided waves. Two end-member fluid flow states are delimited by the timescale of viscous momentum diffusion across the width of the crack:

$$
\alpha^{-1} \equiv w_{0}^{2} / \nu,
$$

where $\nu$ is the kinematic viscosity. At timescales shorter than $\alpha^{-1}$, the fluid velocity is nearly constant across the conduit and the effects of viscosity are confined to narrow boundary layers along the fracture walls. In the opposite limit, at timescales greater than $\alpha^{-1}$, the fluid velocity field has a parabolic, or fully developed, profile. As examples, basaltic magma in a $1 \mathrm{~m}$ wide fracture has $\alpha^{-1} \approx 10 \mathrm{~s}$ and water in a $1 \mathrm{~mm}$ wide fracture has $\alpha^{-1} \approx 1 \mathrm{~s}$. Because seismic frequencies of interest for hydraulic fracturing events are commonly in the range of 1-1000 $\mathrm{Hz}$, a proper description of the hydraulic fracture seismic source must account for boundary layer formation. We restrict attention to low Reynolds number, laminar flows and we assume that the background flow speed in the conduit is negligibly small; all fluid flow in our analysis arises from perturbations to the conduit.

The effects of viscosity have received varying degrees of analysis in previous studies. Many studies have taken the fluid to be inviscid [Aki et al., 1977; Ferrazzini and Aki, 1987; Kumagai and Chouet, 1999, 2000, 2001; Kumagai et al., 2002; Yamamoto and Kawakatsu, 2008]. Chouet [1986, 1988, 1992] and Dunham and Ogden [2012] study the motion of a viscous fluid but assume fully developed flow. Deviations from full flow development have been explored in several recent analytical [Korneev, 2008, 2010; Nakagawa and Korneev, 2014] and numerical [Frehner and Schmalholz, 2010; Frehner, 2014] studies. Several stud- 
ies have also considered turbulent [Hellweg, 2000; Dunham and Ogden, 2012; Tary et al., 2014] and even multiphase flows [Kumagai and Chouet, 2000; Morrissey and Chouet, 2001; Jousset et al., 2004].

Our analysis is most similar to that of Korneev [2008, 2010], but he derives a more general dispersion relation from the linearized Navier-Stokes equation for the fluid and elastic wave equation for the solid. Furthermore, Nakagawa and Korneev [2014], extending the work of Nakagawa and Schoenberg [2007] consider fractures filled with a poroelastic material. That material provides additional stiffness and increases crack wave speeds, particularly at low frequencies. Their framework for analysis also permits consideration of more general relations between pressure gradients along the fracture and the resulting fluid flow. From these various formulations, Korneev [2008, 2010] and Nakagawa and Korneev [2014] take certain limits to examine interesting parts of parameter space, such as when wavelengths are much larger than the crack width. In contrast, we employ various approximations from the outset to arrive at a simplified, approximate set of governing equations. We anticipate that this formulation will be useful for efficient numerical simulation of wave interactions with hydraulic fractures. Solutions to the dispersion relation resulting from our approximate equations reproduce known asymptotic results derived by various authors in relevant limits.

In many cases, we expect that viscous dissipation will control the overall damping of resonant modes. In this limit, we show that observation of the quality factor $Q_{1}$ at the frequency $f_{1}$ provides a second constraint on fracture length and aperture. This constraint complements the relation provided by observations of fundamental resonant frequency $f_{1}$. Together, these two observables provide a means to uniquely constrain fracture geometry 

flow regimes as well as the rigid- and deformable-wall limits.

\section{Governing equations} problem with invariance in the $z$-direction.

These relations arise from the condition that, at resonance for the fundamental mode, wavelengths will be twice the crack length, as discussed in Section 7. In addition, Figures 6-8 present a graphical method for estimating $L$ and $w_{0}$ using $f_{1}$ and $Q_{1}$, based on numerical solution of the dispersion relation. This graphical method provides a more complete solution than (3) and (4) that includes the fully developed and boundary layer

We consider an infinitely long, fluid-filled fracture or conduit that is bounded by two elastic half-spaces (Figure 1). The conduit and all perturbations are taken to be symmetric about the midplane $y=0$. The walls of the conduit are located at $y=-w(x, t)$ and $y=w(x, t)$. The conduit walls are initially planar and parallel, with $w(x, 0)=w_{0}$. Symmetry allows us to restrict attention to $y \geq 0$; we consider the two-dimensional

Direct observations indicate that hydraulic fractures in geologic media are thin in the sense that their lateral extent is often two to four orders of magnitude greater than their cross-sectional opening. This thinness is apparent in aerial images of glacial crevasse fields [Cuffey and Patterson, 2000], outcrops of ancient volcanic dikes [Gudmundsson, 
1983; Walker, 1987; Kavanagh and Sparks, 2011], observations of modern dike extension [Segall et al., 2001; Calais et al., 2008; Biggs et al., 2009], as well as images and cores containing fractures taken from within boreholes [Barton et al., 1995; Gale et al., 2007]. We therefore take the conduit half-width $w_{0}$ to be very narrow in comparison to the fracture-parallel lengthscale $\lambda$ (the crack length or wavelength of guided waves), so that $\epsilon \equiv w_{0} / \lambda \ll 1$. We refer to this condition as the thin-fracture approximation. The condition that $\epsilon \ll 1$ places an upper wavenumber bound on the domain of validity of our analysis. One consequence of this approximation is that in the short-wavelength limit, phase velocities of waves in our model approach the sound speed rather than the slightly slower Scholte wave speed describing propagation along a fluid-solid interface [Stoneley, 1924; Scholte, 1942, 1947].

\subsection{Fluid flow}

We examine small amplitude, symmetric perturbations to a compressible, linear viscous (i.e., Newtonian) fluid that is initially at rest. The fluid has background or unperturbed density $\rho_{0}$ and pressure $p_{0}$ that are both spatially uniform. Neglecting background velocity precludes flow stability analysis as described in several previous studies [Julian, 1994; Balmforth et al., 2005; Rust et al., 2008; Dunham and Ogden, 2012; Sakuraba and Yamauchi, 2014]. 


$$
\frac{1}{\rho_{0}} \frac{\partial \rho}{\partial t}=\frac{1}{K_{f}} \frac{\partial p}{\partial t}
$$

where $K_{f}$ is the fluid bulk modulus. No advective terms arise because the background state has no gradients in fluid density or pressure. As a result of (7) and (8), we conclude that density $\rho$ is also uniform across the width of the conduit.

\subsection{Fluid-solid interface conditions}

Fluid-solid coupling is achieved through interface conditions on the moving wall, $y=$ $w(x, t)$. In this linearized analysis of distubances about a fluid at rest, however, it suffices to enforce approximate interface conditions at the location of the unperturbed conduit wall, $y=w_{0}$.

Force balance at the fluid-solid interface requires that the traction exerted by the elastic solid on the viscous fluid is equal and opposite to that exerted by the fluid on the solid:

$$
p=-\sigma_{y y} \text { on } y=w_{0}
$$




\subsection{Width-averaged description} $x$ direction.

$$
v_{x}=\frac{\partial u_{x}}{\partial t} \text { on } y=w_{0}
$$

and

$$
v_{y}=\frac{\partial u_{y}}{\partial t} \text { on } y=w_{0}
$$
to the velocity at the interface:

$$
\frac{\partial w}{\partial t}=v_{y} \text { on } y=w_{0}
$$

where $\tau$ is the fluid shear stress on the wall, $\sigma_{i j}$ are the components of the stress tensor in the solid, and $O(\epsilon)$ terms are neglected within the linearization. This definition of the wall shear stress $\tau$ is consistent with positive $\tau$ acting to decelerate a flow in the positive

The continuity or no-slip condition states that a particle of fluid that is in contact with the wall remains in contact with the same particle of solid wall material:

where $u_{i}$ are the components of displacement in the solid. The wall-normal velocity condition (12) forbids interpenetration or separation and holds for both viscous and inviscid fluids. Equations (11) and (12) are correct to first order and there is no need to account for deformation-induced rotation of the interface in this linearized analysis.

Finally, the linearized kinematic interface condition relates the motion of the interface

The thin fracture approximation motivates a width-averaged treatment of the fluid. The width-averaged mass balance equation is found by integrating the mass balance (5) 
224

where the width-averaged velocity is

$$
u(x, t)=\frac{1}{w_{0}} \int_{0}^{w_{0}} v_{x}(x, y, t) d y .
$$
material; we return to this subsequently.

The linearized and width-averaged momentum balance is

$$
\rho_{0} \frac{\partial u}{\partial t}=-\frac{\partial p}{\partial x}-\frac{\tau}{w_{0}} .
$$
stresses.

\subsection{Wall elasticity}

We have used the linearized width-averaging operator $w_{0}^{-1} \int_{0}^{w_{0}} d y$. Symmetry permits integrating over the conduit half-width. Nakagawa and Korneev [2014] motivate a similar averaging operation by examining jump conditions across the fracture walls.

The linearized and width-averaged mass balance (14) describes two ways in which the fluid-filled crack accommodates a mass increase $-\partial u / \partial x$. The fluid can either be compressed, as reflected in the $(\partial \rho / \partial t) / \rho_{0}$ term, or the conduit walls can deform, as reflected in the $(\partial w / \partial t) / w_{0}$ term. The relative importance of these effects, which are illustrated in Figure 1, requires consideration of the fluid equation of state and elasticity of the wall

The flow is accelerated by negative pressure gradients and decelerated by positive shear

We consider linear elastic deformation of the conduit walls and limit attention to perturbations having phase velocities much less than the elastic wave speeds. Dunham and Ogden [2012] showed that this phase velocity regime covers most geologic systems of in- 


$$
\hat{F}(k, \omega)=\int_{-\infty}^{\infty} \int_{-\infty}^{\infty} F(x, t) e^{-i(k x-\omega t)} d t d x .
$$

In the transform domain, the relationship between the stresses and displacements on the upper wall of the crack is

$$
\left(\begin{array}{l}
\hat{u}_{x} \\
\hat{u}_{y}
\end{array}\right)=\left(\begin{array}{cc}
-\frac{1-\nu_{s}}{G \mid k} & \frac{1-2 \nu_{s}}{2 i G k} \\
-\frac{1-2 \nu_{s}}{2 i G k} & -\frac{1-\nu_{s}}{G|k|}
\end{array}\right)\left(\begin{array}{l}
\hat{\sigma}_{x y} \\
\hat{\sigma}_{y y}
\end{array}\right) \text { on } y=w_{0} .
$$

The solid shear modulus is $G$ and $\nu_{s}$ is Poisson's ratio. When $k$ is complex, $|k|$ should be interpreted as $\operatorname{sign}(\operatorname{Re} k) k$. We have derived this relationship by taking the quasi-static limit of equation (17) of Ranjith and Rice [2001]. The general inverse dependence on wavenumber indicates that the conduit is more deformable at longer wavelengths. The use of quasi-static elasticity limits us to the regime where the fluid sound speed $c_{0}$ is less than the wall elastic wave speed. More exact theories, such as those developed by Krauklis [1962]; Paillet and White [1982]; Ferrazzini and Aki [1987]; Korneev [2008], and Korneev [2010], account for inertia of the solid and make no assumptions about wavelengths being larger than the crack width, at the expense of a more complex dispersion relation. While those analyses capture the short wavelength behavior more precisely, our approximate treatment is in complete agreement at the longer wavelengths that are the focus of this work.

\section{Viscous compressible flow in a deformable conduit}

In this section we describe the motion of the fluid. There are two limiting behaviors. At low frequencies the fluid velocity profile is parabolic and the flow is fastest in the center of the conduit; the flow is said to be fully developed. At higher frequencies the velocity profile 
$\mathrm{X}-14$ LIPOVSKY AND DUNHAM: RESONANCE OF HYDRAULIC FRACTURES

267

is uniform across most of the conduit, except in narrow boundary layers immediately adjacent to the walls. To describe these phenomena, we first solve for the velocity profile in the cross-conduit or $y$-direction. Using this solution we derive expressions for the widthaveraged velocity $u$ that enters the width-averaged mass balance equation (14) and the wall shear stress $\tau$ that enters the width-averaged momentum balance (16).

We solve the $x$-momentum balance equation with integral transforms. Applying the transform (17) to the momentum balance (6) yields

$$
i \omega \hat{v}_{x}+\nu \frac{d^{2} \hat{v}_{x}}{d y^{2}}=i k \frac{\hat{p}}{\rho_{0}}
$$

where $\nu \equiv \mu / \rho_{0}$ is the kinematic viscosity. Scaling analysis of (19) suggests that the character of the flow will depend on the frequency $\omega$ relative to the viscous timescale $\alpha^{-1}$, as defined in (2). The viscous time $\alpha^{-1}$ is the timescale required for momentum to diffuse across the conduit; $\alpha$ can also be viewed as a viscous damping rate in the fully developed flow limit. The damping ratio $\zeta$ is the ratio of the damping rate $\alpha$ to the angular frequency $\omega:$

$$
\zeta \equiv \frac{\alpha}{\omega}
$$

The parameter $\sqrt{\zeta}$ is the Womersley number, a quantity encountered in cardiovascular mechanics [Womersley, 1955; Barnard et al., 1966].

\subsection{Fluid velocity profile}

The symmetric solution of equation (19) that satisfies the no-slip condition (11) is

$$
\hat{v}_{x}=\hat{a}(y, \omega)\left(-i k \frac{\hat{p}}{\rho_{0}}\right)+\hat{b}(y, \omega)\left(-i \omega \hat{u}_{x}\right),
$$

in which

$$
\hat{a}(y, \omega)=\frac{1}{-i \omega}\left[1-\frac{\cosh \left(\xi y / w_{0}\right)}{\cosh \xi}\right]
$$


and

$$
\hat{b}(y, \omega)=\frac{\cosh \left(\xi y / w_{0}\right)}{\cosh \xi} .
$$

In this solution we have introduced

$$
\xi \equiv \sqrt{-i \frac{w_{0}^{2} \omega}{\nu}},
$$

with branch cut defined so that $\operatorname{Re} \xi \geq 0$ when $\omega$ is complex. Because $\xi^{2}=-i / \zeta$, it is clear that these two parameters represent two ways to express the same information. We retain the use of $\xi$ for notational convenience. The solution for the fluid velocity profile (21) shows that changes in fluid velocity arise from both the fluid pressure and the wallparallel wall motion. The solution (21) matches that of Biot [1956] and Mavko and Nur [1979] in the rigid-wall limit.

In the time domain, the fluid velocity profile is given by a convolution integral of the form

$$
\begin{aligned}
v_{x}(x, y, t)=\int_{-\infty}^{t}\{ & a\left(y, t-t^{\prime}\right)\left[-\frac{1}{\rho_{0}} \frac{\partial p\left(x, t^{\prime}\right)}{\partial x}\right] \\
& \left.+b\left(y, t-t^{\prime}\right)\left[\frac{\partial u_{x}\left(x, t^{\prime}\right)}{\partial t^{\prime}}\right]\right\} \mathrm{d} t^{\prime} .
\end{aligned}
$$

The kernels $a(y, t)$ and $b(y, t)$ may be found by inverting the transform of $(22)$ and $(23)$. For our purposes, however, it is more useful to consider the following asymptotic analyses. The fluid velocity profile (21) has two end-member behaviors: fully developed flow $(\zeta \gg 1)$ and boundary layer flow $(\zeta \ll 1)$.

We begin by describing the velocity for an inviscid fluid $(\zeta=0)$. In this case $\xi \rightarrow \infty$, $a \rightarrow 1 /(-i \omega)$, and $b \rightarrow 0$. In the time domain,

$$
v_{x}(x, t)=-\frac{1}{\rho_{0}} \int_{-\infty}^{t} \frac{\partial p\left(x, t^{\prime}\right)}{\partial x} d t^{\prime} .
$$


$\mathrm{X}-16$ LIPOVSKY AND DUNHAM: RESONANCE OF HYDRAULIC FRACTURES

The fluid velocity profile is constant across the conduit and horizontal wall motion plays no role in the fluid motion. The fluid is uniformly accelerated by the pressure gradient.

The high frequency limit for a viscous fluid $(\zeta \ll 1)$ is characterized by the formation of narrow boundary layers near the wall. The width of the boundary layer is $\sim \sqrt{\nu / \omega}$. Outside of the boundary layers the velocity profile is nearly constant and matches the inviscid solution (26). The transfer functions take the asymptotic forms

$$
\begin{aligned}
& \hat{a}(y, \omega)=\frac{1}{-i \omega}\left\{1-\exp \left[-\xi\left(1-\frac{y}{w_{0}}\right)\right]\right\}, \\
& \hat{b}(y, \omega)=\exp \left[-\xi\left(1-\frac{y}{w_{0}}\right)\right] .
\end{aligned}
$$

The fluid velocity profile is

$$
\begin{aligned}
v_{x}(x, y, t) & =\int_{-\infty}^{t} \operatorname{erf}\left(\frac{1-y / w_{0}}{\sqrt{4 \alpha\left(t-t^{\prime}\right)}}\right)\left(-\frac{1}{\rho_{0}} \frac{\partial p\left(x, t^{\prime}\right)}{\partial x}\right) d t^{\prime} \\
& +\int_{-\infty}^{t} \operatorname{erfc}\left(\frac{1-y / w_{0}}{\sqrt{4 \alpha\left(t-t^{\prime}\right)}}\right)\left(\frac{\partial^{2} u_{x}\left(x, t^{\prime}\right)}{\partial t^{\prime 2}}\right) d t^{\prime}
\end{aligned}
$$

In the low frequency, fully developed flow limit $(\zeta \gg 1)$

$$
\begin{aligned}
& \hat{a}(y, \omega)=\frac{w_{0}^{2}}{2 \nu}\left(1-\frac{y^{2}}{w_{0}^{2}}\right), \\
& \hat{b}(y, \omega)=1 .
\end{aligned}
$$

Inverting these transfer functions shows that the velocity field has a parabolic profile:

$$
v_{x}(x, y, t)=\frac{w_{0}^{2}}{2 \mu}\left(1-\frac{y^{2}}{w_{0}^{2}}\right)\left(-\frac{\partial p}{\partial x}\right)+\frac{\partial u_{x}}{\partial t},
$$

and we see that the velocity only depends on the instantaneous pressure gradient and wall-parallel wall velocity. 


\subsection{Width-averaged fluid velocity and wall shear stress}

$$
T \equiv \frac{\tanh \xi}{\xi}
$$

In the boundary layer limit, $\zeta \ll 1$ and to leading order in $\xi^{-1}$,

$$
T \approx \frac{1}{\xi}=\sqrt{i \zeta}
$$

In the fully developed limit, $\zeta \gg 1$ and

$$
T=1-\frac{1}{3} \xi^{2}+\cdots \approx 1-\frac{1}{3 i \zeta} .
$$

The wall shear stress $\tau$ defined by equation (10) is found by differentiating the velocity field (21) and evaluating the result at $y=w_{0}$. The result is

$$
\hat{\tau}=w_{0} \rho_{0} T\left(-\frac{i k \hat{p}}{\rho_{0}}\right)+i \omega w_{0} \rho_{0} T\left(-i \omega \hat{u}_{x}\right) .
$$

We combine (34) and (38) to eliminate pressure $p$ from the expression for wall shear stress $\tau$ :

$$
\hat{\tau}=-i \omega w_{0} \rho_{0} \frac{T}{1-T}\left(\hat{u}+i \omega \hat{u}_{x}\right) .
$$

In the fully developed flow limit (39) takes the time domain limit

$$
\tau=3 \frac{\mu}{w_{0}}\left(u-\frac{\partial u_{x}}{\partial t}\right),
$$

which shows that the wall shear stress only depends on the difference between the average fluid velocity and the horizontal wall velocity. We have used the limit that $T /(1-T) \approx 3 i \zeta$ 
from (37). Neglecting horizontal wall motion, as we later find is justified, results in the same drag law, $\tau=3 \mu u / w_{0}$, that was used by Dunham and Ogden [2012]. Dunham and Ogden [2012], however, used this drag law for all $\zeta$, including in the $\zeta \ll 1$ limit where it is not appropriate.

In the boundary layer limit, the wall shear stress is history dependent. We eliminate pressure in (34) and (39), and with the help of (36), we find the convolution integral

$$
\tau(x, t)=\frac{\mu}{\sqrt{4 \pi}} \int_{-\infty}^{t} \frac{\partial u\left(x, t^{\prime}\right) / \partial t^{\prime}-\partial^{2} u_{x}\left(x, t^{\prime}\right) / \partial t^{\prime 2}}{\sqrt{\nu\left(t-t^{\prime}\right)}} d t^{\prime} .
$$

We note that shear stress does not depend on the fracture width in the boundary layer limit. This is expected because the fluid shear is confined to a narrow region near the wall.

\subsection{Scaling Relations}

Several scaling relationships arise between $\tau, u$, and $p$ that may be useful in calculating the magnitudes of these fields. In Appendix B we show that the horizontal motion of the wall has a negligible role in wave motion. For this reason we neglect its contribution in this section.

The fully developed flow and boundary layer limits each have characteristic average velocities. In the fully developed flow limit this characteristic velocity is

$$
u_{F D} \equiv \frac{w_{0}^{2}}{3 \mu}\left|-\frac{\partial p}{\partial x}\right|
$$

which reflects the dominant balance in (6) between the pressure gradient driving the flow and viscous resistance. In this limit, inertial effects are negligible and the average velocity is out of phase with pressure (Figure 2). 
In contrast, the characteristic average velocity in the boundary layer limit is

$$
u_{B L} \equiv \frac{1}{\omega \rho_{0}}\left|-\frac{\partial p}{\partial x}\right|
$$

now reflecting the dominant balance between the pressure gradient and fluid inertia. The presence of boundary layers confines the effects of viscosity to a thin region near the conduit wall and, for time-harmonic motions, allows the central part of the flow to maintain a velocity that is in phase with the pressure perturbation, as for an inviscid fluid (Figure 2). In the boundary layer limit the average velocity is independent of viscosity and conduit width.

In the fully developed flow limit, the wall shear stress scales as

$$
\tau_{F D} \equiv w_{0}\left|-\frac{\partial p}{\partial x}\right|
$$

and in the boundary layer limit as

$$
\tau_{B L} \equiv \sqrt{\frac{\nu}{\omega}}\left|-\frac{\partial p}{\partial x}\right|
$$

As before in (41), it is useful to eliminate pressure $p$ to state the scaling relationship between $\tau$ and $u$; for fully developed flow,

$$
\tau_{F D}=\frac{3 \mu}{w_{0}} u_{F D}
$$

In the boundary layer limit the corresponding relationship is

$$
\tau_{B L}=\mu \frac{u_{B L}}{\sqrt{\nu / \omega}}
$$

For a linear viscous fluid, the shear stress in a viscous fluid is the product of the shear strain rate and the dynamic viscosity. In the fully developed flow limit, momentum has diffused across the entire width of the conduit, and the shear strain rate is $\sim u_{F D} / w_{0}$. 

(1)

In the boundary layer limit, the shear strain rate is $\sim u_{B L} / \sqrt{\nu / \omega}$, where $\sqrt{\nu / \omega}$ is the momentum diffusion length.

We emphasize that the scaling relationships (42)-(47) are not exact expressions for the evolution of velocities and shear stresses. Rather, they are estimates of the amplitude response to a harmonic perturbation.

\section{Wave dispersion relation}

Our goal is to describe the motions that arise from the coupled fluid-elastic system. Seeking solutions with $e^{i(k x-\omega t)}$ dependence to the homogeneous equations results in a dispersion relation $D(k, \omega)=0$ that relates $k$ and $\omega$.

The governing equations are the width-averaged conservation of mass (14) and $x$ momentum (16), the equations of elasticity (18), and the fluid drag law (39). Together, these relations form a homogeneous system of equations:

$$
\left(\begin{array}{ccccc}
\frac{\omega}{K_{f}} & 0 & -k & \frac{\omega}{w_{0}} & 0 \\
0 & 1 & i k w_{0} T & 0 & \omega^{2} w_{0} \rho_{0} T \\
-i k & -1 / w_{0} & i \omega \rho_{0} & 0 & 0 \\
-\frac{1-\nu_{s}}{G|k|} & -\frac{1-2 \nu_{s}}{2 G k} & 0 & 1 & 0 \\
-\frac{1-2 \nu_{s}}{2 i G k} & \frac{1-M_{s}}{G|k|} & 0 & 0 & 1
\end{array}\right)\left(\begin{array}{c}
\hat{p} \\
\hat{\tau} \\
\hat{u} \\
\hat{w} \\
\hat{u}_{x}
\end{array}\right)=\mathbf{0} .
$$

In this matrix equation, the equation of state (8) was used to eliminate density $\rho$ and the kinematic boundary condition (13) was used to eliminate $v_{y}$. The system (48) has nontrivial solutions only when the determinant of the coefficient matrix, the characteristic equation, vanishes. Without approximation, the characteristic equation is 
413

in which $T=\tanh (\xi) / \xi, G^{*}=G /\left(1-\nu_{s}\right)$ and $G^{\prime}=G /\left(1-2 \nu_{s}\right)$. Ignoring $O\left(\epsilon^{2}\right)$ terms, as discussed in Appendix B, yields the simplified dispersion relation

$$
\begin{aligned}
D(k, \omega) & \equiv\left(\frac{\tanh \xi}{\xi}-1\right)\left(\frac{c_{0}}{\omega / k}\right)^{2}+1+\frac{K_{f}}{G^{*}|k| w_{0}} \\
& =0
\end{aligned}
$$

Several nondimensional parameters appear in the dispersion relation (50). We have already discussed the nondimensional viscous damping parameter $\zeta$ in equation (20), which enters (50) through $\xi$. The ratio of the restoring forces from fluid compressibility and elastic wall deformation is [Dunham and Ogden, 2012]

$$
\Lambda \equiv \frac{K_{f}}{G^{*}|k| w_{0}} .
$$

Understood as a ratio of wavelengths (or wavenumbers), the parameter $\Lambda=\lambda / \lambda_{e l}$ defines a characteristic elastic coupling length scale

$$
\lambda_{e l} \equiv 2 \pi \frac{G^{*} w_{0}}{K_{f}}
$$

and associated wavenumber $k_{e l} \equiv 2 \pi / \lambda_{e l}$. When $\Lambda \gg 1$, waves have restoring force from the elasticity of the conduit walls. When $\Lambda \ll 1$, waves have restoring force from fluid compressibility. It will sometimes be useful to characterize the restoring force regime by frequency rather than by wavenumber. For this purpose, we define the corresponding elastic coupling frequency,

$$
\omega_{e l} \equiv k_{e l} c_{0} \equiv \frac{K_{f} c_{0}}{G^{*} w_{0}} .
$$

This characteristic elastic coupling frequency gives rise to the nondimensional frequency,

$$
\Omega \equiv \frac{\omega}{\omega_{e l}} .
$$


We use $\Omega$ when studying waves with real-valued frequency $\omega$ and $\Lambda$ when studying waves with real-valued wavenumber $k$.

We nondimensionalize the wavenumber by the wavenumber of a sound wave with angular frequency $\omega$ :

(1)

$$
K \equiv \frac{k}{\omega / c_{0}} .
$$

We note that

$$
\Lambda=\frac{1}{\Omega|K|} .
$$

The dispersion relation (50) may be written using these nondimensional parameters:

its solutions depend only on the dimensionless wavenumber $K$, and the two parameters $\Omega$, and $\zeta$, the latter by way of the dependence $T(\zeta)$. In the rest of this work we describe the wave motion that arises from this relation.

\section{Wave behavior with real frequency}

We consider two types of excitation of hydraulic fracture guided waves. In the first scenario waves have a real-valued frequency $\omega$. This real-frequency model of wave propagation results in a spatial quality factor and is well suited to describe the spatial decay of perturbations that propagate along a fracture, away from a constant-frequency source [e.g., Montagna and Gonnermann, 2013]. In Section 6 we consider the contrasting case of a wave propagating with real-valued wavenumber $k$. Such a real-wavenumber model of wave propagation results in a temporal quality factor and is well suited to describe the temporal decay of resonant modes of a finite-length hydraulic fracture. 

damping from seismic radiation.

The normalized wave phase velocity is

$$
\frac{c}{c_{0}} \equiv \frac{1}{\operatorname{Re} K}
$$
confinement of viscous dissipation to narrow boundary layers.

Because such waves travel in a quasi-one-dimensional wave guide there is no decrease in amplitude due to geometrical spreading. Instead, hydraulic fracture guided waves experience amplitude decay through viscous dissipation in the fluid. We also neglect

Phase velocity and attenuation, obtained by numerical solution of the dispersion relation (57), are plotted in Figure 3. Waves are anomalously dispersed in the sense that phase velocity is an increasing function of frequency. Attenuation, in contrast, is a decreasing function of frequency. Diminished attenuation at high frequencies is due to the 
Two important transitions occur as $\omega$ is varied: the transition from sound waves to crack waves and the transition from fully developed flow to boundary layer flow. The first is associated with the frequency $\omega_{e l}$ and the second with $\alpha$. Upon choosing one of these to nondimensionalize $\omega$, the only remaining parameter governing wave behavior is $\omega_{e l} / \alpha$.

These two transitions divide the parameter space into four quadrants. In the remainder of this section we present closed-form analytic solutions for each of these end-member wave behaviors. We first describe waves in a rigid-walled conduit $\left(\Omega^{-1}=0\right)$. We then describe waves in a highly deformable conduit $(\Omega \ll 1)$. For each of these two scenarios we discuss boundary layer $(\zeta \ll 1)$ and fully developed $(\zeta \gg 1)$ flow. We begin with the simpler case where viscosity is neglected and $\zeta=0$.

Seismic observations of resonating, fluid-filled cracks in geologic media often exist in a part of parameter space where perturbations propagate as crack waves with boundary layer flow. These waves have low attenuation because they are in the boundary layer limit; they have high fluid-solid coupling in the sense that a pressure perturbation results in a greater wall deformation in the crack wave limit than in the sound wave limit. Boundary layer crack waves exist in the frequency range $\alpha<\omega<\omega_{e l}$. Asymptotic limits of the phase velocity and attenuation in this range are given in Section 5.2.2. For a $1 \mathrm{~m}$ dike filled with basaltic melt in crustal rock, this range is approximately $6 \times 10^{-3} \mathrm{~Hz}<f<10 \mathrm{~Hz}$ (Table 4 ), where $f \equiv \omega /(2 \pi)$. For a $1 \mathrm{~mm}$ water-filled fracture in ice this range is approximately $0.2 \mathrm{~Hz}<\omega<2 \times 10^{5} \mathrm{~Hz}$.

Most geologic systems have $\omega_{e l} / \alpha \gg 1$, but our results do not depend on this condition. Whenever $\omega_{e l}>\alpha$ waves can occur as fully developed crack waves, boundary layer crack waves, and boundary layer sound waves. When $\omega_{e l}>\alpha$, fully developed sound waves 


\subsubsection{Inviscid case}

In the simplest scenario a rigid-walled conduit contains an inviscid fluid $(\zeta=0)$. Because $T=0$, the dispersion relation equation (57) reduces to

$$
K=1
$$

or $k=\omega / c_{0}$. These are unattenuated sound waves that propagate with the nondispersive phase velocity equal to the fluid sound speed $c_{0}$.

\subsubsection{Boundary layer limit}

We now consider effects of viscosity. In the high frequency, $\zeta \ll 1$ limit, we approximate $T$ as in (36). The approximate dispersion relation is

$$
K=\left(1+i \sqrt{\frac{\zeta}{8}}\right)
$$

The phase velocity is again $c=c_{0}$, as in the inviscid case. Attenuation is

$$
\frac{1}{2 Q}=\sqrt{\frac{\zeta}{8}}=\frac{1}{2 w_{0}} \sqrt{\frac{\nu}{2 \omega}} .
$$

\subsubsection{Fully developed flow limit}

At lower frequencies, $\zeta \gg 1$ and the dispersion relation is

$$
K=(1+i) \sqrt{\frac{3}{2} \zeta},
$$

where we have used (37). In this limit, damped sound waves have a dispersive phase velocity

$$
\frac{c}{c_{0}}=\sqrt{\frac{2}{3 \zeta}}=w_{0} \sqrt{\frac{2}{3} \frac{\omega}{\nu}} .
$$

Attenuation is constant, with

$$
\frac{1}{2 Q}=1
$$


This attenuation result is not plotted in Figure 3 because, as discussed previously, fully developed sound waves do not occur when $\alpha<\omega_{e l}$.

\subsection{Crack waves and the deformable conduit limit}

Perturbations to the fracture result in crack waves when $\Omega^{-1} \gg 1$. As before, we consider two limits of the fluid flow: boundary layer $(\zeta \ll 1)$ and fully developed $(\zeta \gg 1)$.

\subsubsection{Inviscid case}

We initially neglect viscosity by taking $\zeta=0$. The dispersion relation is

$$
K=\Omega^{-1 / 3}
$$

The phase velocity is

$$
c=\left(\omega \frac{G^{*} w_{0}}{K_{f} c_{0}}\right)^{1 / 3},
$$

as stated in equation (1). Because $K_{f}=\rho_{0} c_{0}^{2}, c$ depends only on fluid density $\rho_{0}$ and not on the compressibility $K_{f}$. Following Chouet [1986] we refer to such waves as crack waves. Several recent studies refer to such waves as either Krauklis or Stoneley guided waves [Korneev, 2008, 2010; Frehner and Schmalholz, 2010; Frehner, 2014], although other names have also been used [Korneev et al., 2012].

\subsubsection{Boundary layer limit}

When $\zeta \ll 1$, the dispersion relation is

$$
K=\Omega^{-1 / 3}\left(1+\sqrt{\frac{\zeta}{9}}\right) .
$$

Boundary layer crack waves have the same phase velocity as undamped crack waves (68); their attenuation remains independent of the elastic coupling parameter $\Omega$,

$$
\frac{1}{2 Q}=\frac{1}{3 w_{0}} \sqrt{\frac{\nu}{2 \omega}},
$$


562

The attenuation (70) differs only from the boundary layer sound wave attenuation (63)

by a small constant factor that amounts to $\approx 6 \%$ difference in $1 / 2 Q$.

\subsubsection{Fully developed flow limit}

The dispersion relation for $\zeta \gg 1$ is

$$
K=i^{1 / 3}\left(\frac{3 \zeta}{\Omega}\right)^{1 / 3}=\frac{\sqrt{3}+i}{2}\left(\frac{3 \zeta}{\Omega}\right)^{1 / 3} .
$$

The phase velocity,

$$
c=\frac{2}{\sqrt{3}}\left(\frac{\Omega c_{0}^{3}}{3 \zeta}\right)^{1 / 3}=\frac{2 w_{0}}{\sqrt{3}}\left(\frac{1}{3} \frac{\omega^{2} G^{*}}{\eta}\right)^{1 / 3}
$$

is less than the undamped crack wave velocity (68). This expression matches that given in Korneev [2008] in his (37). The phase velocity (72) applies regardless of whether $\alpha>\omega_{e l}$ or $\omega_{e l}>\alpha$. Attenuation is given by

$$
\frac{1}{2 Q}=\frac{1}{\sqrt{3}}
$$

The dispersion relation (71) is cubic and therefore has three roots. The first two roots have the same properties but propagate in opposite directions. The third root does not correspond to a wave-like solution because it has zero phase velocity. When this mode is excited by a perturbation, it will decay rapidly with distance and will not cause disturbances to propagate or travel away from the perturbation.

\section{Wave behavior with real wavenumber}

In Section 5 we considered waves with real-valued frequency $\omega$. This wave model results in a complex-valued wavenumber. In this section we consider the complementary case of waves with real-valued wavenumber $k$ and complex-valued frequency, $\omega=\omega_{R}+i \omega_{I}$. This model is well suited to describe the temporal decay of the resonance of a finite-length fracture in terms of both its frequency and decay rate or temporal quality factor. We 
which is the same as equation (57) except that $k$ is now considered to be a real-valued parameter. We recall that $\xi=\sqrt{-i \omega / \alpha}$, with branch cut defined so that $\operatorname{Re} \xi \geq 0$.

Solutions to the dispersion relation, which are plotted in Figures 4-5, are characterized by two nondimensional parameters. The restoring force parameter $\Lambda$ distinguishes between sound waves $(\Lambda \ll 1)$ and crack waves $(\Lambda \gg 1)$. We note that equation $(57)$ used the restoring force parameter $\Omega$ because for real-valued $\omega, \Omega$ is also real-valued. In this 
608

section we use $\Lambda \equiv k_{e l} / k$ (51) to express the restoring force ratio. Additionally, damping of real-wavenumber waves is characterized by the parameter $\alpha /\left(k c_{0}\right)$. This parameter is qualitatively similar to $\zeta$ as defined in $(20)$.

\subsection{Inviscid case}

In the special case of an inviscid fluid, $\tanh \xi / \xi \rightarrow 0$, and the dispersion relation (76) is

$$
\frac{\omega}{k c_{0}}=\sqrt{\frac{1}{1+\Lambda}}
$$

This solution to the dispersion relation is always real-valued and so its solutions are undamped. When $\Lambda \ll 1$, (77) describes sound waves; when $\Lambda \gg 1$, waves have phase velocity

$$
c=c_{0} / \sqrt{\Lambda}=\sqrt{\frac{G^{*}|k| w_{0}}{\rho_{0}}}
$$

The real- $k$ sound wave and crack wave solutions of (77) and (78) are the same as the real- $\omega$ sound wave and crack wave solutions of (61) and (67). The sound wave and crack wave phase velocities are plotted as a function of wavenumber in Figure 4.

\subsection{Boundary layer limit}

To account for a small amount of viscous damping, we carry out an asymptotic analysis by considering a correction to the inviscid solution (77). We write the complex frequency $\omega=\omega_{0}+\omega_{1}$. We take $\omega_{0}=k c_{0}(1+\Lambda)^{-1 / 2}$ and substitute $\omega=\omega_{0}+\omega_{1}$ into (76). We then treat $\alpha(1+\Lambda)^{1 / 2} /\left(k c_{0}\right)$ as a small parameter and solve for $\omega_{1}$. The result is the boundary layer limit dispersion relation:

$$
\frac{\omega}{k c_{0}}=\sqrt{\frac{1}{1+\Lambda}}\left[1-(i+1) \sqrt{\frac{\alpha}{8 k c_{0}} \sqrt{1+\Lambda}}\right] .
$$


The general phase velocity and temporal attenuation that result from this dispersion relation are

$$
c=c_{0} \sqrt{\frac{1}{1+\Lambda}}\left(1-\sqrt{\frac{\alpha}{8 k c_{0}} \sqrt{1+\Lambda}}\right),
$$

and

$$
\frac{1}{2 Q}=\sqrt{\frac{\alpha}{8 k c_{0}} \sqrt{1+\Lambda}} .
$$

Equations (80) and (81) are used to derive (3) and (4).

\subsection{Fully developed flow and the cutoff wavenumber}

The dispersion relationship in the fully developed flow limit is found by taking the limit of $(76)$ when $\omega / \alpha \ll 1$. We use the Taylor series

$$
\frac{1}{1-T} \approx \frac{3}{\xi^{2}} \frac{1}{1-\frac{2}{5} \xi^{2}+\cdots} \approx \frac{3}{\xi^{2}}+\frac{6}{5}
$$

consistent with (37), and arrive at the dispersion relation

$$
\frac{6}{5}\left(\frac{\omega}{k c_{0}}\right)^{2}+3 i \frac{\alpha}{k c_{0}}\left(\frac{\omega}{k c_{0}}\right)-\frac{1}{1+\Lambda}=0
$$

This dispersion relation is the same as that considered by Dunham and Ogden [2012] except for the appearance of the prefactor of $6 / 5$. The difference results from the uneven use of fluid inertia by Dunham and Ogden [2012], who retained fluid inertia in their widthaveraged momentum balance equation (our equation 16) but not in solving for the fluid drag relationship (i.e., our equation 19). Equation (83) is quadratic in $\omega /\left(k c_{0}\right)$ and so has the simple solution

$$
\frac{\omega}{k c_{0}}=\frac{5}{4}\left[-i \frac{\alpha}{k c_{0}} \pm \sqrt{\frac{8}{15} \frac{1}{1+\Lambda}-\left(\frac{\alpha}{k c_{0}}\right)^{2}}\right]
$$

\subsubsection{Long wavelength limit}


At long wavelengths there exists a cutoff wavenumber below which perturbations have zero phase velocity. The cutoff wavelength occurs when the radical in (84) vanishes.

Setting the radical equal to zero and isolating the wavenumber results in

$$
k_{c} \equiv\left(\frac{15}{8} \frac{k_{e l} \alpha^{2}}{c_{0}^{2}}\right)^{1 / 3}=\left(\frac{15}{8} \frac{\mu^{2}}{G^{*} \rho_{0} w_{0}^{5}}\right)^{1 / 3}
$$

for the most relevant, $\Lambda \gg 1$, limit.

The dispersion relation (83) may then be rewritten, in this limit, using the cutoff wavenumber,

$$
\frac{\omega}{\alpha}=\frac{5}{4}\left[-i \pm \sqrt{\left(\frac{k}{k_{c}}\right)^{3}-1}\right] .
$$

At wavenumbers near but greater than the cutoff wavenumber, the phase velocity and attenuation are

$$
c=\frac{5}{4} \frac{\alpha}{k} \sqrt{\frac{k}{k_{c}}-1}
$$

and

$$
\frac{1}{2 Q}=\left(\frac{k}{k_{c}}-1\right)^{-1 / 2} .
$$

The phase velocity vanishes at $k=k_{c}$. This attenuation expression shows that $1 / 2 Q$ diverges as $k \rightarrow k_{c}$, which is a consequence of the oscillation frequency Re $\omega \rightarrow 0$ as the decay rate $\operatorname{Im} \omega$ remains finite.

\subsubsection{Low frequency limit}

Waves with real wavenumber exist at arbitrarily low frequencies but the wavelength approaches a finite value (the cut-off wavelength) as frequency approaches zero. To quantify the seismic frequencies associated with hydraulic fracture resonance at some real wavenumber (which would be related to the fracture length) we examine the frequency dependence of waves with real wavenumbers. This is done by stating the phase veloc- 
670

ity and temporal attenuation of such waves with explicit reference to the frequency of oscillation $\omega_{R}$.

Near the cutoff wavenumber the phase velocity is

$$
c=\frac{\omega_{R}}{k_{c}},
$$

where we have used $k=k_{c}$ in (75). Note that as $k \rightarrow k_{c}, \omega_{R} \rightarrow 0$ and hence $c \rightarrow 0$. We refer to this type of wave motion as real-wavenumber damped crack waves. These waves occur at frequencies $\omega_{R}<\alpha$ and they propagate more slowly than their counterpart with real frequency (72). The phase velocity is plotted in Figure 3 as a function of $\omega_{R}$.

The temporal attenuation is

$$
\frac{1}{2 Q}=\frac{5}{4} \frac{\alpha}{\omega_{R}}
$$

where we have used (86) in (74). We note that spatial and temporal attenuation do not coincide in the low frequency, fully developed flow limit. This is in contrast to the high frequency, boundary layer limit, where spatial attenuation as a function of real $\omega$ and temporal attenuation as a function of $\omega_{R}$ coincide. Figure 5 shows temporal attenuation as a function of frequency $\omega_{R}$ and Figure $3 \mathrm{~b}$ shows temporal attenuation as a function of frequency $\omega$.

\subsection{Attenuation in the boundary layer and fully developed flow limits}

Several previous analyses have analyzed attenuation in the fully developed flow limit [Chouet, 1986; Balmforth et al., 2005; Dunham and Ogden, 2012]. In this limit and for real wavenumbers, the attenuation relation is (90) [Dunham and Ogden, 2012]. This attenuation relation, however, has been used at frequencies $\omega>\alpha$ where it is not strictly valid. In this limit, the correct attenuation is described by the boundary-layer relations 
(81) and (70). Near the frequency $\omega=\alpha$, these two relations give the same value for $1 / 2 Q$.

At higher frequencies, the difference between these two relations grows. For $\omega_{e l} / \alpha \sim 10^{4}$, as in the example of a $1 \mathrm{~m}$ wide dike filled with basalt shown in Figure 3b, the fully developed flow attenuation relation underestimates the actual attenuation by about two orders of magnitude.

\section{Inferring hydraulic fracture geometry using resonant modes}

While our theory has been developed for infinitely long conduits, we can apply it in an approximate manner to study the resonant vibrations of a finite length crack that are thought to explain many long period seismic events. This is done for the two-dimensional problem involving a hydraulic fracture with length $L$, but the general relations should apply also to the more realistic three-dimensional problem, provided that the crack is approximately equidimensional.

\subsection{Resonance condition}

Resonance occurs when pairs of counter-propagating waves along the waveguide constructively interfere at certain wavelengths related to the crack length and boundary conditions at the crack tips. We restrict attention to a crack with closed tips, for which $u=0$ at both ends, though our theory could be generalized to cracks connected to some form of reservoir at one or both ends.

For a closed fracture, a solution to the governing equations and boundary conditions $(u=0$ at $x=0$ and $x=L)$ is $u \propto \sin (n \pi x / L)$ and $p-p_{0} \propto \cos (n \pi x / L)$ for $n=1,2, \ldots$ The corresponding wavelengths are thus $\lambda_{n}=2 L / n$ and resonant frequencies are

$$
f_{n}=c\left(\lambda_{n}\right) / \lambda_{n}
$$


Resonances from nondispersive waves have $f_{n} / f_{1}=n$, implying that overtones appear at integer multiples of the fundamental $(n=1)$ mode. In contrast, dispersion results in noninteger spacing between resonant frequencies. Specifically, for waves in the crack wave regime, combining the crack wave phase velocity (78) with (91) gives

$$
\frac{f_{n}}{f_{1}}=n^{3 / 2}
$$

The frequency spacing between successive peaks therefore increases. This could serve as a useful diagnostic for deciding if some observed resonances are indeed associated with fluid-filled cracks.

\subsection{Geometry estimation}

Each resonant frequency $f_{n}$ has an associated temporal quality factor, $Q_{n}$. Together, the resonance frequency and the quality factor constitute two seismically observable attributes of a long period seismic event. The resonance frequencies and quality factors, for specified material properties, may be thought of as functions of the fracture length and aperture. Using the resonance condition (91) we write the temporal attenuation and characteristic frequency of the $n^{\text {th }}$ mode as

$$
\begin{gathered}
Q_{n}=Q_{n}\left(w_{0}, L\right), \\
f_{n}=f_{n}\left(w_{0}, L\right) .
\end{gathered}
$$

We obtain these functions numerically by solving the dispersion relation (76) with wavenumber $k_{n}=n \pi / L$. The inverse problem is then to estimate $w_{0}$ and $L$ from a data set of $Q_{n}$ and $f_{n}$.

Here we limit attention to the lowest frequency $(n=1)$ mode; although this mode has lower $Q$ than higher modes, its decay time will be the longest, making it the most 

and (4).

\subsection{Analysis of data} seismic amplitude or seismic power is plotted. and (93), over a range of $w_{0}$ and $L$ in Figures 6-8. In the sound wave limit the lines of resonant frequency (94) are vertical because the phase velocity and thus frequency do not depend on fracture width in this limit. In the boundary layer crack wave limit, asymptotic expressions for the characteristic frequency and quality factor are given by equations (3)

We analyze published data from several studies (Table 1); the results are plotted in Figures 6-8. Our analysis of these data is in reasonable agreement with previous estimates of fracture length $L$. Most previous studies, however, have not attempted to simultaneously constrain fracture length and width. Instead, previous work has simply assumed some width thought to be reasonable for the system under study. In some of the data, multiple spectral peaks are observed. In this study we analyze only the fundamental mode. However, we note that if multiple spectral peaks are observed, an optimization problem could be carried out to find the best geometry or geometries of one or several fractures.

When analyzing data, we estimate the quality factor in one of two ways. When analyzing spectral peaks, by assuming decay according to $e^{-f_{1} t /\left(2 Q_{1}\right)}$, the quality factor may be calculated as $Q_{1}=f_{1} / \Delta f$, where $\Delta f$ is the width, in frequency units, of the spectral peak at the level of $1 / \sqrt{2}$ of the maximum amplitude. Alternatively, when seismograms are available, we may count the number of oscillations that occur before an amplitude decay to $4 \%$ of the signal's initial value. There is some uncertainty in using previously published spectra because published spectrograms may not state a color scale or whether 
We interpret the seismic signal of Anandakrishnan and Alley [1997] near the grounding line of the Kamb Ice Stream, Antarctica as due to a fracture with an aperture $\sim 1 \mathrm{~cm}$. This estimate is consistent with other observations of basal water layer thickness[Kamb, 2001; Schroeder et al., 2014]. All of the other glacier data that we analyze suggest fracture apertures on the order of 1 to $3 \mathrm{~mm}$. This is notable because our estimates of fracture lengths show much greater variability: from about $1 \mathrm{~m}$ at Bakaninbreen to $12 \mathrm{~m}$ at Cotopaxi Glacier. Assuming the cracks open when pressure $p_{0}$ exceeds a remote compressive stress $\sigma$ with linear elastic response of the solid, as opposed to melting, we can estimate the excess pressure as $p_{0}-\sigma \sim G^{*} w_{0} / L$. Except for Bakaninbreen, estimated $p_{0}-\sigma$ are a few MPa. In contrast, the elastic-opening estimate for Bakaninbreen is $50 \mathrm{MPa}$. This unreasonably large value likely suggests that the fracture opened by melting instead of elastic deformation.

Many volcano LP events are striking because of their quality factors as large as $Q_{1} \sim 1000$. Large $Q$ requires low damping and therefore small $\alpha \equiv \nu / w_{0}^{2}$ relative to the wave frequency $\omega$. We find that in some cases, even for the relatively low viscosity of basaltic melts, our model requires wide conduits to explain volcanic LP events as due to the resonance of fractures filled with magma. The waveform of Kumagai and Chouet [1999] from Galeras Volcano, for example, requires a conduit on the order of $60 \mathrm{~m}$ in width for a fracture filled with a basaltic magma ( $\mu \sim 100 \mathrm{~Pa}$ s). Galeras Volcano has a dominantly andesitic composition and we expect the viscosity of melt to be much higher than that of basalt. Fractures filled with high viscosity andesitic melts $\left(\mu \sim 10^{5} \mathrm{~Pa} \mathrm{~s}\right)$, however, produce the observed quality factors only for conduits that are so wide as to 
be geologically unrealistic. It may be the case that basaltic intrusions are present in this generally andesitic volcano [Eichelberger and Izbekov, 2000].

Hydrothermal fluid-filled fractures are another possible source of volcanic LP events. We carry out calculations to estimate fracture length and width assuming this composition, and generally find that smaller fractures satisfy the data (Figure 6). At Galeras Volcano, for example, the fracture width and length are estimated to be $40 \mathrm{~cm}$ and $80 \mathrm{~m}$. The general interpretation of volcanic LP events originating from the volcano-hydrothermal system is consistent with a large literature on this topic [Kumagai et al., 2005; Waite et al., 2008; Matoza and Chouet, 2010; Arciniega-Ceballos et al., 2012].

In the reservoir setting, Tary et al. [2014] interpret a seismic event to be due to a source fracture with $L=15-30 \mathrm{~m}$; our estimate is $L=7 \mathrm{~m}$ and $2 w_{0}=8 \mathrm{~mm}$. The discrepancy in fracture length is due to a mistake in equation (A1) of Tary et al. [2014], where a factor of $2 \pi$ is necessary in the denominator of the radical because $f=\omega / 2 \pi$.

In the Fenton Hill geothermal site Ferrazzini et al. [1990] estimate a fracture to have in-plane dimensions of $3 \mathrm{~m}$ and $1 \mathrm{~m}$ and width of $3 \mathrm{~mm}$, respectively. Our estimated length is $1 \mathrm{~m}$ and width is $1 \mathrm{~mm}$. Ferrazzini et al. [1990] neglected the viscosity of water in their analysis; instead all attenuation is from seismic radiation.

We also point out that contrary to our prediction of nonequally spaced resonant frequencies, the overtones in the Tary et al. [2014] and Anandakrishnan and Alley [1997] data sets are at integer multiples of the fundamental frequency. It is therefore possible that these seismograms are not caused by hydraulic fracture resonances as idealized in our work. 


\subsection{Uncertainties and limitations}

Our fracture geometry estimation method has several uncertainties and limitations arising from various approximations. While for certain approximations the estimates is length and width can be viewed as either upper or lower bounds, taken together, it is not possible to classify our estimates as strict upper or lower bounds.

Our use of a two-dimensional model probably results in a reasonable description of roughly equidimensional fractures. But for fractures with large aspect ratio, such as dikes extending laterally for many kilometers, the elastic response will be determined by the shorter height dimension rather than the length and crack wave propagation will become nondispersive at long wavelengths. Our description requires modification for such problems.

In addition, we calculate phase velocity and attenuation for sinusoidal waves propagating along an infinitely long channel. A finite length crack will have increased elastic stiffness, which will alter the shape of the eigenfunctions and shift resonant frequencies (especially of the lowest modes) to higher values. With correcting for this additional stiffness, we will underestimate length.

Similarly, we neglect variations in width, such as roughness of fracture walls and the tapering of width to zero at the crack tips. The latter has been considered, in an approximate way, by Tary et al. [2014], who suggest that it will decrease resonant frequencies. The decrease in width will also alter flow and will eventually lead to fully developed flow and increased attenuation in some region near the crack tips. We estimate that many fractures have mm-scale aperture, which suggests that the realistically rough fracture walls may be in contact at points of geometric irregularity; this might increase the stiffness of 
the fracture in the wall-normal direction and increase the crack wave phase velocities, as shown by Nakagawa and Korneev [2014].

We do not include all possible sources of attenuation, such as damping from seismic radiation, multiphase fluid interactions, poroelastic effects, and other sources described by various authors [Kumagai and Chouet, 2000; Morrissey and Chouet, 2001; Jousset et al., 2004; Nakagawa and Korneev, 2014]. Since both $w_{0}$ and $L$ decrease with increasing $Q_{1}$, this approximation results makes our estimates upper bounds on both $w_{0}$ and $L$.

The existence of the cutoff wavelength poses an additional limitation to this method. It is possible that the first observable resonant mode might not correspond to the $\lambda_{1}=2 L$ mode, but instead to the first higher mode having wavelength shorter than the cutoff wavelength. When a higher mode is incorrectly interpreted as the fundamental mode, length will be underestimated.

We have also assumed that fractures are open and that velocity vanishes at the two crack tips. But if a fracture connects to some fluid reservoir or the atmosphere at one end, then its resonant frequencies will be altered.

Lastly, we note that data window length might prevent adequate measurement of the quality factor. In order to observe a quality factor $Q$, a data window of duration $\sim Q / f_{1}$ is required. Of the data we have analyzed here, only the data of Anandakrishnan and Alley [1997] are potentially affected by this limitation. The data windows of Anandakrishnan and Alley [1997] have a fixed duration of $12 \mathrm{~s}$, which for $75 \mathrm{~Hz}$ oscillations results in a lower-bound estimate of $Q_{1} \sim 500$. 


\section{Moment-characteristic frequency scaling}

Long period seismic events due to hydraulic fracture resonant modes have unique scaling relations that might be useful when interpreting statistics of catalogs of such events. Such catalogs have been collected, for example, by Anandakrishnan and Alley [1997], Aki and Ferrazzini [2000], Kumagai et al. [2002], Okubo and Wolfe [2008], West et al. [2010], and Power et al. [2013]. Such analysis may also be relevant to glacial crevassing events [Neave and Savage, 1970; Walter et al., 2008; Walter et al., 2010; Walter et al., 2013; Röösli et al., 2014].

The source process of an LP event is the oscillation of the walls of a hydraulic fracture. The characteristic amplitude of oscillatory width changes is $\Delta w \equiv w-w_{0}$, and for a roughly equidimensional fracture of dimension $L$ this gives rise to the seismic moment

$$
M_{0} \sim G^{*} L^{2} \Delta w .
$$

Whereas seismic sources with a static offset have seismic moment proportional to the low frequency asymptote of the displacement spectra, we note that oscillatory seismic sources have seismic moment related to the maximum amplitude of the fundamental spectral peak.

These oscillations in width are caused by pressure changes $\Delta p \equiv p-p_{0}$ in the crack, which are related by linear elasticity (18):

$$
\Delta p \sim G^{*} \Delta w / L .
$$

We can envision no physical process that would introduce any systematic dependence of $\Delta p$ on crack length $L$. We henceforth take $\Delta p$ to be constant. This assumption is analogous to the constant stress drop assumption for earthquakes. 
For resonant cracks, the dominant frequency is related to the crack length by way of the phase velocity: $f_{1} \sim c / L$. Combining (91), (95), and (96) results in a scaling law relating seismic moment and oscillation frequency:

$$
M_{0} \sim\left(\frac{c}{f_{1}}\right)^{3} \Delta p
$$

In the nondispersive sound wave limit, (97) gives $M_{0} \propto f_{1}^{-3}$. However, in the crack wave limit, where $c$ is given by (1), this relationship becomes

$$
M_{0} \sim\left(\frac{G^{*} w_{0} \Delta p}{\rho_{0}}\right) f_{1}^{-2} .
$$

The scaling law (98) contrasts with the well-known scaling for earthquakes, $M_{0} \propto f_{1}^{-3}$, due to the dispersive nature of crack waves.

Alternatively, (97) may be written to express the pressure change $\Delta p$ as a function of moment and characteristic frequency:

$$
\Delta p \sim \frac{M_{0} \rho_{0}}{G^{*} w_{0}} f_{1}^{2} .
$$

This relationship (97) is depicted for two systems in Figure 9, where the phase velocity $c$ is found numerically by solving (76). The different scaling between $M_{0}$ and $f_{1}$ for shear fractures versus resonating hydraulic fractures may be useful in differentiating between these two processes in situations where both may plausibly occur.

\section{Conclusions}

We have described wave motion along a thin hydraulic fracture wave guide with an emphasis on viscous attenuation. A central result of this analysis is a method to estimate hydraulic fracture width and length from seismic observations (Figure 6). We conclude by highlighting several assumptions made in this work and by suggesting how future work may lift several of these assumptions to better represent seismic observations. 
downloaded from the Incorporated Research Institutions for Seismology (IRIS) website, iris.edu on April 4, 2014.

\section{Appendix A: Derivation of the governing equations}

The goal of this appendix is to simplify the mass and momentum balance equations using scaling arguments. In two spatial dimensions, the linearized equations are

$$
\begin{aligned}
\frac{\partial \rho}{\partial t}+\rho_{0} \frac{\partial v_{x}}{\partial x}+\rho_{0} \frac{\partial v_{y}}{\partial y} & =0 \\
\rho_{0} \frac{\partial v_{x}}{\partial t}+\frac{\partial p}{\partial x} & =\mu\left(2 \frac{\partial^{2} v_{x}}{\partial x^{2}}+\frac{\partial^{2} v_{x}}{\partial y^{2}}+\frac{\partial^{2} v_{y}}{\partial x \partial y}\right) \\
\rho_{0} \frac{\partial v_{y}}{\partial t}+\frac{\partial p}{\partial y} & =\mu\left(\frac{\partial^{2} v_{y}}{\partial x^{2}}+2 \frac{\partial^{2} v_{y}}{\partial y^{2}}+\frac{\partial^{2} v_{x}}{\partial x \partial y}\right) \\
\frac{1}{\rho_{0}} \frac{\partial \rho}{\partial t} & =\frac{1}{K_{f}} \frac{\partial p}{\partial t}
\end{aligned}
$$

We have included the fluid equation of state (A4) for completeness.

We nondimensionalize these governing equations by introducing the following dimensionless quantities:

$$
\begin{aligned}
& v_{x}^{\prime}=v_{x} / c_{x}, \\
& v_{y}^{\prime}=v_{y} / c_{y}, \\
& x^{\prime}=x / \lambda,
\end{aligned}
$$$$
y^{\prime}=y / w_{0},
$$$$
t^{\prime}=t /(\lambda / c)
$$ 
where $c_{x}$ and $c_{y}$ are characteristic particle velocities, $\lambda$ is the wavelength, and pressure and density fluctuations are made nondimensional with the wave impedance $\rho_{0} c$,

$$
\begin{aligned}
\left(p-p_{0}\right)^{\prime} & =\frac{p-p_{0}}{\rho_{0} c c_{x}}, \\
\left(\rho-\rho_{0}\right)^{\prime} & =\frac{\rho-\rho_{0}}{\rho_{0} c_{x} / c} .
\end{aligned}
$$

The governing equations have eight dimensional quantities $\left(c_{x}, c_{y}, c, c_{0}, \lambda, w_{0}, \rho_{0}\right.$, and $\left.\mu\right)$ to represent three dimensions (length, time, and mass). The system is therefore described by five nondimensional parameters. Since the system is linear and homogeneous, one of these parameters is a nondimensional perturbation amplitude. We choose the four remaining parameters to be

$\begin{aligned} c / c_{0}, & \\ \gamma & \equiv \frac{c_{y}}{c_{x}}, \\ \epsilon & \equiv \frac{w_{0}}{\lambda}, \\ \zeta_{*} & \equiv \frac{\alpha}{c / \lambda} .\end{aligned}$

The damping parameter $\zeta_{*}$ is qualitatively similar to $\zeta \equiv \alpha / \omega$ defined in the main text (20) in that $\zeta_{*}$ and $\zeta$ both reflect the amount of viscous damping during one wave period.

The linearized Navier-Stokes equations may be written using these nondimensional fields as

$$
\begin{aligned}
\epsilon\left(\frac{c}{c_{0}}\right)^{2} \frac{\partial p^{\prime}}{\partial t^{\prime}}+\epsilon \frac{\partial v_{x}^{\prime}}{\partial x^{\prime}}+\gamma \frac{\partial v_{y}^{\prime}}{\partial y^{\prime}}=0 \\
\frac{\partial v_{x}^{\prime}}{\partial t^{\prime}}+\frac{\partial p^{\prime}}{\partial x^{\prime}}=\zeta_{*}\left(2 \epsilon^{2} \frac{\partial^{2} v_{x}^{\prime}}{\partial x^{\prime 2}}+\frac{\partial^{2} v_{x}^{\prime}}{\partial y^{\prime 2}}+\epsilon \gamma \frac{\partial^{2} v_{y}^{\prime}}{\partial x^{\prime} \partial y^{\prime}}\right),
\end{aligned}
$$$$
\epsilon \gamma \frac{\partial v_{y}^{\prime}}{\partial t^{\prime}}+\frac{\partial p^{\prime}}{\partial y^{\prime}}=\zeta_{*}\left(\gamma \epsilon^{3} \frac{\partial^{2} v_{y}^{\prime}}{\partial x^{\prime 2}}+2 \gamma \epsilon \frac{\partial^{2} v_{y}^{\prime}}{\partial y^{\prime 2}}+\epsilon^{2} \frac{\partial^{2} v_{x}^{\prime}}{\partial x^{\prime} \partial y^{\prime}}\right) \text {. }
$$

We have used the fluid equation of state (8) to eliminate $\rho$ in the mass balance (A16). 
We take our fundamental scaling assumptions to be

$$
\epsilon \ll 1 \text { and } \gamma \ll 1 \text {, }
$$

but we make no assumption about the relative sizes of $\epsilon$ and $\gamma$. The choice of $\epsilon \ll 1$ is the thin fracture assumption, as discussed in the main text. The choice of $\gamma \ll 1$ is due to our focus on long wavelength, symmetric perturbations. For crack waves involving negligible changes in density, the mass balance (A16) implies that $\gamma \sim \epsilon$. The choice of $\gamma \ll 1$ also precludes the antisymmetric mode of Ferrazzini and Aki [1987]. With this scaling there are no generally negligible terms in the mass balance equation. In the momentum balance equations we neglect the $O\left(\epsilon^{2}\right)$ and $O(\epsilon \gamma)$ terms. Reverting to dimensional form yields the approximate linearized Navier-Stokes equations,

$$
\begin{aligned}
\frac{\partial \rho}{\partial t}+\rho_{0} \frac{\partial v_{x}}{\partial x}+\rho_{0} \frac{\partial v_{y}}{\partial y} & =0 \\
\rho_{0} \frac{\partial v_{x}}{\partial t}-\mu \frac{\partial^{2} v_{x}}{\partial y^{2}}+\frac{\partial p}{\partial x} & =0 \\
\frac{\partial p}{\partial y} & =0 .
\end{aligned}
$$

\section{Appendix B: Approximating the dispersion relation}

In this appendix we demonstrate that the dispersion relation (49), which we restate here as

$$
\begin{array}{r}
(T-1) \frac{k^{2} c_{0}^{2}}{\omega^{2}}+\frac{K_{f}}{G^{*} w_{0}|k|}+1 \\
+T\left[\frac{K_{f} \omega^{2} \rho_{0}}{4\left(G^{\prime}\right)^{2} k^{2}}-\frac{K_{f} \omega^{2} \rho_{0}}{\left(G^{*}\right)^{2}|k|^{2}}+(1-2 T) \frac{k^{2} K_{f} w_{0}}{G^{*}|k|}-\frac{\omega^{2} \rho_{0} w_{0}}{G^{*}|k|}\right] \\
=0
\end{array}
$$

may be approximated by the first three terms, as stated in (50). The first three terms are order unity or larger, so it will suffice to show that the remaining terms in brackets are 
and the third scales as

$$
\frac{K_{f} \omega^{2} \rho_{0}}{G^{2} k^{2}} \sim\left(\frac{K_{f}}{G}\right)^{2}\left(\frac{c}{c_{0}}\right)^{2}
$$

We have assumed that $G \sim G^{*} \sim G^{\prime}$ and $|k| \sim k$. The second scales as

$$
\frac{K_{f}}{G} k w_{0} \sim\left(\frac{K_{f}}{G}\right) \epsilon
$$

First we show these terms are negligible in the sound wave limit. We assume that phase velocities in the fluid-elastic system are bounded by the sound wave speed, $\omega / k \leq c_{0}$, which can be verified a posteriori. Wavenumbers are thus bounded by

$$
k \geq \frac{\omega}{c_{0}}>\frac{\omega_{e l}}{c_{0}}=\frac{K_{f}}{w_{0} G^{*}}
$$

because sound waves occur only when $\omega>\omega_{e l}$. Rearranging (B5) gives

$$
K_{f} / G^{*} \lesssim \epsilon
$$

which means that thin fractures $(\epsilon \ll 1)$ host sound waves only when $K_{f} / G \ll 1$. Using (B6) and $c \sim c_{0}$ we find that$$
\left(\frac{K_{f}}{G}\right)^{2}\left(\frac{c}{c_{0}}\right)^{2} \sim \epsilon^{2}
$$$$
\left(\frac{K_{f}}{G}\right) \epsilon \sim \epsilon^{2}
$$$$
\left(\frac{c}{c_{0}}\right)^{2}\left(\frac{K_{f}}{G}\right) \epsilon \sim \epsilon^{2} \text {. }
$$

Each term is $O\left(\epsilon^{2}\right)$ and thus may be neglected. 
We next show that these terms are negligible in the crack wave limit. In this case,

$\Lambda \gg 1$ and

$$
\left(\frac{c}{c_{0}}\right)^{2} \sim \frac{1}{\Lambda}
$$

The terms (B2)-(B4) become

$$
\begin{aligned}
\left(\frac{K_{f}}{G}\right)^{2}\left(\frac{c}{c_{0}}\right)^{2} \sim & \left(\frac{K_{f}}{G}\right) \epsilon \sim \epsilon^{2} \Lambda, \\
\left(\frac{K_{f}}{G}\right) \epsilon & \sim \epsilon^{2} \Lambda, \\
\left(\frac{c}{c_{0}}\right)^{2}\left(\frac{K_{f}}{G}\right) \epsilon & \sim \epsilon^{2} .
\end{aligned}
$$

These terms are therefore negligible relative to terms of $O(1)$ and of $O(\Lambda)$.

The simplified dispersion relation is therefore

$$
D(k, \omega) \equiv(T-1) \frac{k^{2} c_{0}^{2}}{\omega^{2}}+\frac{K_{f}}{G^{*} w_{0}|k|}+1=0
$$

as claimed in (50).

\section{References}

Aki, K. (1984), Evidence for magma intrusion during the Mammoth Lakes earthquakes of May 1980 and implications of the absence of volcanic (harmonic) tremor, J. Geophys. Res., 89(B9), 7689-7696, doi:10.1029/JB089iB09p07689.

Aki, K., and V. Ferrazzini (2000), Seismic monitoring and modeling of an active volcano for prediction, J. Geophys. Res., 105(B7), 16,617-16,640, doi:10.1029/2000JB900033.

Aki, K., M. Fehler, and S. Das (1977), Source mechanism of volcanic tremor: Fluid-driven crack models and their application to the 1963 Kilauea eruption, J. Volcanol. Geotherm. Res., 2(3), 259-287, doi:10.1016/0377-0273(77)90003-8. 
Anandakrishnan, S., and R. Alley (1997), Tidal forcing of basal seismicity of ice stream

C, West Antarctica, observed far inland, J. Geophys. Res., 102(B7), 15,183-15,196.

Arciniega-Ceballos, A., P. Dawson, and B. Chouet (2012), Source characterization of LP seismicity at Popocatepetl Volcano, Mexico, in Geological Society of America Abstracts with Programs, vol. 44, p. 20.

Balmforth, N. J., R. V. Craster, and A. C. Rust (2005), Instability in flow through elastic conduits and volcanic tremor, J. Fluid Mech., 527, 353-377, doi: 10.1017/S0022112004002800.

Bame, D., and M. Fehler (1986), Observations of long period earthquakes accompanying hydraulic fracturing, Geophys. Res. Lett., 13, 149-152, doi:10.1029/GL013i002p00149.

Barnard, A., W. Hunt, W. Timlake, and E. Varley (1966), A theory of fluid flow in compliant tubes, Biophys. J., 6(6), 717-724, doi:10.1016/S0006-3495(66)86690-0.

Barton, C. A., M. D. Zoback, and D. Moos (1995), Fluid flow along potentially active faults in crystalline rock, Geology, 23(8), 683-686, doi:10.1130/00917613(1995)023<0683:FFAPAF > 2.3.CO;2.

Biggs, J., F. Amelung, N. Gourmelen, T. H. Dixon, and S.-W. Kim (2009), InSAR observations of 2007 Tanzania rifting episode reveal mixed fault and dyke extension in an immature continental rift, Geophys. J. Int., 179(1), 549-558, doi:10.1111/j.1365246X.2009.04262.x.

Biot, M. A. (1956), Theory of propagation of elastic waves in a fluid-saturated porous solid. I. Low-frequency range, J. Acoust. Soc. Am., 28(2), 168-178, doi:10.1121/1.1908241.

Calais, E., N. d'Oreye, J. Albaric, A. Deschamps, D. Delvaux, J. Déverchere, C. Ebinger, R. W. Ferdinand, F. Kervyn, A. S. Macheyeki, et al. (2008), Strain accommodation 
by slow slip and dyking in a youthful continental rift, East Africa, Nature, 456(7223), 783-787, doi:10.1038/nature07478.

Chouet, B. (1986), Dynamics of a fluid-driven crack in three dimensions by the finite difference method, J. Geophys. Res., 91 (B14), 13,967-13,992, doi:10.1029/JB091iB14p13967.

Chouet, B. (1988), Radiation properties and implications for the source of longperiod events and harmonic tremor, J. Geophys. Res., 93(B5), 4375-4400, doi: 10.1029/JB093iB05p04375.

Chouet, B. (1992), A seismic model for the source of long-period events and harmonic tremor, in Volcanic Seismology, edited by P. Gasparini, R. Scarpa, and K. Aki, no. 3 in IAVCEI Proceedings in Volcanology, pp. 133-156, Springer, Berlin, Germany.

Chouet, B. A. (1996), Long-period volcano seismicity: its source and use in eruption forecasting, Nature, 380, 309-316, doi:10.1038/380309a0.

Chouet, B. A., and R. S. Matoza (2013), A multi-decadal view of seismic methods for detecting precursors of magma movement and eruption, J. Volcanol. Geotherm. Res., 252, 108-175, doi:10.1016/j.jvolgeores.2012.11.013.

Chouet, B. A., R. A. Page, C. D. Stephens, J. C. Lahr, and J. A. Power (1994), Precursory swarms of long-period events at Redoubt Volcano (1989-1990), Alaska: Their origin and use as a forecasting tool, J. Volcanol. Geotherm. Res., 62(1), 95-135, doi:10.1016/03770273(94)90030-2.

Creyts, T. T., and C. G. Schoof (2009), Drainage through subglacial water sheets, J. Geophys. Res., 114(F4), 4008, doi:10.1029/2008JF001215.

Cuffey, K., and W. Patterson (2000), The Physics of Glaciers, 4 ed., Elsevier, Burlington, USA. 
Dunham, E. M., and D. E. Ogden (2012), Guided waves along fluid-filled cracks in elastic solids and instability at high flow rates, J. of Appl. Mech., 79(3), a031,020, doi: 10.1115/1.4005961.

Eichelberger, J. C., and P. E. Izbekov (2000), Eruption of andesite triggered by dyke injection: contrasting cases at Karymsky Volcano, Kamchatka and Mt Katmai, Alaska, Philos. Trans. R. Soc. of Lond., 358(1770), 1465-1485, doi:10.1098/rsta.2000.0599.

Ferrazzini, V., and K. Aki (1987), Slow waves trapped in a fluid-filled infinite crack: Implication for volcanic tremor, J. Geophys. Res., 92(B9), 9215-9224, doi: 10.1029/JB092iB09p09215.

Ferrazzini, V., B. Chouet, M. Fehler, and K. Aki (1990), Quantitative analysis of longperiod events recorded during hydrofracture experiments at Fenton Hill, New Mexico, J. Geophys. Res., 95(B13), 21,871-21,884, doi:10.1029/JB095iB13p21871.

Frehner, M. (2014), Krauklis wave initiation in fluid-filled fractures by seismic body waves, Geophys., 79(1), T27-T35, doi:10.1190/geo2013-0093.1.

Frehner, M., and S. M. Schmalholz (2010), Finite-element simulations of Stoneley guidedwave reflection and scattering at the tips of fluid-filled fractures, Geophys., 75(2), T23T36, doi:10.1190/1.3340361.

Freund, L. (1971), The oblique reflection of a Rayleigh wave from a crack tip, Int. J. Solid. Struct., 7(9), 1199-1210, doi:10.1016/0020-7683(71)90061-8.

Gale, J., R. Reed, and J. Holder (2007), Natural fractures in the Barnett Shale and their importance for hydraulic fracture treatments, AAPG Bulletin, 91(4), 603-622, doi:10.1306/11010606061. 
$\mathrm{X}-52$ LIPOVSKY AND DUNHAM: RESONANCE OF HYDRAULIC FRACTURES

Groenenboom, J., and J. Falk (2000), Scattering by hydraulic fractures: Finite-difference modeling and laboratory data, Geophys., 65(2), 612-622, doi:10.1190/1.1444757.

Gudmundsson, A. (1983), Form and dimensions of dykes in eastern Iceland, Tectonophys., 95(34), 295 - 307, doi:10.1016/0040-1951(83)90074-4.

Hellweg, M. (2000), Physical models for the source of Lascar's harmonic tremor, J. Volcanol. Geotherm. Res., $101(1), 183-198$.

Jousset, P., J. Neuberg, and A. Jolly (2004), Modelling low-frequency volcanic earthquakes in a viscoelastic medium with topography, Geophys. J. Int., 159(2), 776-802.

Julian, B. R. (1994), Volcanic tremor: Nonlinear excitation by fluid flow, J. Geophys. Res., 99, 11,859-11,877, doi:10.1029/93JB03129.

Kamb, B. (2001), Basal zone of the West Antarctic ice streams and its role in lubrication of their rapid motion, Antarct. Res. Ser., 77, 157-199, doi:10.1029/AR077p0157.

Kavanagh, J., and R. S. J. Sparks (2011), Insights of dyke emplacement mechanics from detailed 3D dyke thickness datasets, J. Geol. Soc., 168(4), 965-978, doi:10.1144/001676492010-137.

Korneev, V. (2008), Slow waves in fractures filled with viscous fluid, Geophys., 73(1), N1-N7, doi:0.1190/1.2802174.

Korneev, V. (2010), Low-frequency fluid waves in fractures and pipes, Geophys., 75(6), N97-N107, doi:10.1190/1.3484155.

Korneev, V., G. Goloshubin, B. Kashtan, A. Bakulin, V. Troyan, G. Maximov, L. Molotkov, M. Frehner, S. Shapiro, and R. Shigapov (2012), Krauklis wave - Half a century after, in 5th EAGE Saint Petersburg International Conference \& Exhibition Expanded Abstract, pp. 2-5. 
Krauklis, P. (1962), About some low frequency oscillations of a liquid layer in elastic medium, Prikl. Mat. Mek., 26, 1111-1115, in Russian.

Kumagai, H., and B. A. Chouet (1999), The complex frequencies of long-period seismic events as probes of fluid composition beneath volcanoes, Geophys. J. Int., 138(2), F7F12, doi:10.1046/j.1365-246X.1999.00911.x.

Kumagai, H., and B. A. Chouet (2000), Acoustic properties of a crack containing magmatic or hydrothermal fluids, J. Geophys. Res., 105(B11), 25,493-25, doi: 10.1029/2000JB900273.

Kumagai, H., and B. A. Chouet (2001), The dependence of acoustic properties of a crack on the resonance mode and geometry, Geophys. Res. Lett., 28(17), 3325-3328, doi: 10.1029/2001GL013025.

Kumagai, H., B. A. Chouet, and M. Nakano (2002), Temporal evolution of a hydrothermal system in Kusatsu-shirane volcano, Japan, inferred from the complex frequencies of long-period events, J. Geophys. Res., 107(B10), ESE-9, doi:10.1029/2001JB000653.

Kumagai, H., B. A. Chouet, and P. B. Dawson (2005), Source process of a long-period event at Kilauea Volcano, Hawaii, Geophys. J. Int., 161 (1), 243-254, doi:10.1111/j.1365246X.2005.02502.x.

Matoza, R. S., and B. A. Chouet (2010), Subevents of long-period seismicity: Implications for hydrothermal dynamics during the 2004-2008 eruption of Mount St. Helens, J. Geophys. Res., 115(B12), n/a-n/a, doi:10.1029/2010JB007839.

Mavko, G. M., and A. Nur (1979), Wave attenuation in partially saturated rocks, Geophys., 44(2), 161-178, doi:10.1190/1.1440958. 
$X-54$ LIPOVSKY AND DUNHAM: RESONANCE OF HYDRAULIC FRACTURES

Métaxian, J.-P., S. Araujo, M. Mora, and P. Lesage (2003), Seismicity related to the glacier of Cotopaxi Volcano, Ecuador, Geophys. Res. Lett., 30(9), doi:10.1029/2002GL016773.

Montagna, C. P., and H. M. Gonnermann (2013), Magma flow between summit and Pu'u'ō'ō at Kīlauea Volcano, Hawai'i, Geochem. Geophys. Geosyst., doi: 10.1002/ggge.20145.

Morrissey, M., and B. Chouet (2001), Trends in long-period seismicity related to magmatic fluid compositions, J. Volcanol. Geotherm. Res., 108(1), 265-281, doi:10.1016/S03770273(00)00290-0.

Nakagawa, S., and V. A. Korneev (2014), Effect of fracture compliance on wave propagation within a fluid-filled fracture, J. Acoust. Soc. Am., 135(6), 3186-3197, doi: 10.1121/1.4875333.

Nakagawa, S., and M. A. Schoenberg (2007), Poroelastic modeling of seismic boundary conditions across a fracture, J. Acoust. Soc. Am., 122(2), 831-847, doi: $10.1121 / 1.2747206$.

Neave, K. G., and J. C. Savage (1970), Icequakes on the Athabasca Glacier, J. Geophys. Res., $75(8)$, 1351-1362, doi:10.1029/JB075i008p01351.

Okubo, P. G., and C. J. Wolfe (2008), Swarms of similar long-period earthquakes in the mantle beneath Mauna Loa Volcano, J. Volcanol. Geotherm. Res., 178(4), 787-794, doi:10.1016/j.jvolgeores.2008.09.007.

Paillet, F., and J. White (1982), Acoustic modes of propagation in the borehole and their relationship to rock properties, Geophys., 47(8), 1215-1228, doi:10.1190/1.1441384.

Power, J., S. Stihler, B. Chouet, M. Haney, and D. Ketner (2013), Seismic observations of Redoubt Volcano, Alaska - 1989-2010 and a conceptual model of the Redoubt magmatic 
system, J. Volcanol. Geotherm. Res., 259, 31-44, doi:10.1016/j.jvolgeores.2012.09.014.

Ranjith, K., and J. Rice (2001), Slip dynamics at an interface between dissimilar materials, J. Mech. Phys. Solids, 49(2), 341-361, doi:10.1016/S0022-5096(00)00029-6.

Röösli, C., F. Walter, S. Husen, L. Andrews, M. Lüthi, G. Catania, and E. Kissling (2014), Sustained seismic tremors and icequakes detected in the ablation zone of the Greenland ice sheet, J. Glacio., 60(221), doi:10.3189/2014JoG13J210.

Rubin, A. (1995), Propagation of magma-filled cracks, Ann. Rev. Earth Planet. Sci., 23, 287-336, doi:10.1146/annurev.ea.23.050195.001443.

Rust, A., N. Balmforth, and S. Mandre (2008), The feasibility of generating low-frequency volcano seismicity by flow through a deformable channel, Geol. Soc. Lond., 307(1), 4556, doi:10.1144/SP307.4.

Sakuraba, A., and H. Yamauchi (2014), Linear stability of plane poiseuille flow in an infinite elastic medium and volcanic tremors, Earth, Planets and Space, 66(1), 1-24.

Scholte, J. (1942), On the Stoneley wave equation, in Proc. K. Ned. Akad. Wet, vol. 45, pp. 20-25.

Scholte, J. (1947), The range of existence of Rayleigh and Stoneley waves, Geophys. J. Int., 5 (s5), 120-126, doi:10.1111/j.1365-246X.1947.tb00347.x.

Schroeder, D., D. Blankenship, R. Raney, and C. Grima (2014), Estimating subglacial water geometry using radar bed echo specularity: Application to Thwaites Glacier, West Antarctica, Geosci. Remote Sens. Lett., IEEE, 12(3), 443-447, doi: 10.1109/LGRS.2014.2337878.

Segall, P., P. Cervelli, S. Owen, M. Lisowski, and A. Miklius (2001), Constraints on dike propagation from continuous GPS measurements, J. Geophys. Res., 106(B9), 19,301- 
19,317, doi:10.1029/2001JB000229.

Sparks, R. S. J., J. Biggs, and J. W. Neuberg (2012), Monitoring volcanoes, Science, 335(6074), 1310-1311, doi:10.1126/science.1219485.

Stoneley, R. (1924), Elastic waves at the surface of separation of two solids, Proc. R. Soc. Lond., pp. 416-428.

Stuart, G., T. Murray, A. Brisbourne, P. Styles, and S. Toon (2005), Seismic emissions from a surging glacier: Bakaninbreen, Svalbard, Ann. Glacio., 42(1), 151-157, doi: $10.3189 / 172756405781812538$.

Tary, J., M. Baan, and D. Eaton (2014), Interpretation of resonance frequencies recorded during hydraulic fracturing treatments, J. Geophys. Res., 119, 1295-1315, doi:10.1002/2013JB010904.

Waite, G. P., B. A. Chouet, and P. B. Dawson (2008), Eruption dynamics at Mount St. Helens imaged from broadband seismic waveforms: Interaction of the shallow magmatic and hydrothermal systems, J. Geophys. Res., 113(B2), doi:10.1029/2007JB005259.

Walker, G. P. L. (1987), The dike complex of Koolau Volcano, Oahu: internal structure of a Hawaiian rift zone, in Volcanism in Hawaii, vol. 2, edited by R. W. Decker, T. L. Wright, and P. H. Stauffer, United States Geological Survey, USGS Professional Paper 1350.

Walter, F., N. Deichmann, and M. Funk (2008), Basal icequakes during changing subglacial water pressures beneath Gornergletscher, Switzerland, J. Glacio., 54(186), 511521.

Walter, F., D. S. Dreger, J. F. Clinton, N. Deichmann, and M. Funk (2010), Evidence for Near-Horizontal Tensile Faulting at the Base of Gornergletscher, a Swiss Alpine Glacier, 
Bull. Seismol. Soc. Am., 100, 458-472, doi:10.1785/0120090083.

Walter, F., P. Dalban Canassy, S. Husen, and J. F. Clinton (2013), Deep icequakes: What happens at the base of alpine glaciers?, J. Geophys. Res., 118(3), 1720-1728, doi:10.1002/jgrf.20124.

West, M. E., C. F. Larsen, M. Truffer, S. O'Neel, and L. LeBlanc (2010), Glacier microseismicity, Geology, 38(4), 319-322, doi:10.1130/G30606.1.

Winberry, J., S. Anandakrishnan, and R. Alley (2009), Seismic observations of transient subglacial water-flow beneath MacAyeal Ice Stream, West Antarctica, Geophys. Res. Lett., 36, 11,502, doi:10.1029/2009GL037730.

Womersley, J. R. (1955), Method for the calculation of velocity, rate of flow and viscous drag in arteries when the pressure gradient is known, J Physio., 127(3), 553-563.

Yamamoto, M., and H. Kawakatsu (2008), An efficient method to compute the dynamic response of a fluid-filled crack, Geophys. J. Int., 174(3), 1174-1186, doi:10.1111/j.1365246X.2008.03871.x. 
Table 1. Seismic Observations and Inferred Geometries

\begin{tabular}{|c|c|c|c|c|c|c|}
\hline \multirow{2}{*}{\multicolumn{2}{|c|}{ Study }} & \multirow[b]{2}{*}{ Location } & \multicolumn{2}{|c|}{ Observation } & \multicolumn{2}{|c|}{ Model $^{\mathrm{a}}$} \\
\hline & & & $f_{1}(\mathrm{~Hz})$ & $Q_{1}$ & $L(\mathrm{~m})$ & $2 w_{0}(\mathrm{~m})$ \\
\hline 1. & $\begin{array}{l}\text { Anandakrishnan } \\
\text { and Alley [1997] }\end{array}$ & Kamb Ice Stream & 75 & 500 & 2 & 0.04 \\
\hline 2. & $\begin{array}{l}\text { Winberry et al. } \\
{[2009]^{\mathrm{c}}}\end{array}$ & $\begin{array}{l}\text { MacAyeal Ice } \\
\text { Stream }\end{array}$ & 3 & 3 & 7 & 0.002 \\
\hline 3. & $\begin{array}{l}\text { Métaxian et al. } \\
{[2003]^{\mathrm{d}}}\end{array}$ & Cotopaxi Glacier & 1 & 1 & 12 & 0.002 \\
\hline 4. & Stuart et al. $[2005]^{\mathrm{e}}$ & Bakaninbreen & 75 & 30 & 0.9 & 0.003 \\
\hline 5. & West et al. $[2010]^{\mathrm{f}}$ & Bering Glacier & 6 & 3 & 4 & 0.001 \\
\hline 6. & $\begin{array}{l}\text { Kumagai and } \\
\text { Chouet }[1999]^{\mathrm{g}}\end{array}$ & $\begin{array}{l}\text { Kusatsu-Shirane } \\
\text { Volcano }\end{array}$ & 8 & 400 & $30-60$ & $0.1-20$ \\
\hline 7. & $\begin{array}{l}\text { Kumagai and } \\
\text { Chouet [1999] }\end{array}$ & Galeras Volcano & 3 & 1000 & $80-200$ & $0.4-60$ \\
\hline 8. & $\begin{array}{l}\text { Kumagai and } \\
\text { Chouet [1999] }\end{array}$ & Kilauea Volcano & 1.5 & 40 & $50-200$ & $0.02-4$ \\
\hline 9. & $\begin{array}{l}\text { Kumagai and } \\
\text { Chouet [1999] }\end{array}$ & Redoubt Volcano & 6 & 40 & $20-50$ & $0.01-2$ \\
\hline 10. & $\begin{array}{l}\text { Ferrazzini et al. } \\
{[1990]^{\mathrm{h}}}\end{array}$ & $\begin{array}{l}\text { Fenton Hill } \\
\text { Geothermal Site }\end{array}$ & 112 & 20 & 1 & 0.001 \\
\hline 11. & Tary et al. $[2014]^{\mathrm{i}}$ & $\begin{array}{l}\text { Cardium } \\
\text { Formation }\end{array}$ & 17 & 40 & 7 & 0.008 \\
\hline
\end{tabular}

a The range of values presented for volcanic LP events indicates estimates based on a waterfilled fracture (smaller value) and a fracture filled with basaltic magma (larger value). Length and width estimates for cryospheric systems are calculated for a water-filled fracture in ice and estimates for reservoirs are calculated for water-filled fractures in rock.

b Seismogram from this study downloaded from iris.edu. A representative event is used from data recorded at station XF.DN3S on Day 335 of 1995 at 03:05:45.9120 local time. The quality factor was computed from the spectrum of the entire 12 second trigger window.

c Data from their Figure 2c. Quality factor reported by Winberry et al. [2009].

d Data from their Figure 4. Quality factor reported by Métaxian et al. [2003].

e Data from their Figure 5a. Quality factor measured from spectrum.

f Data from their Figure 2a, "Low Frequency" trace. Quality factor measured from seismogram.

g Data from their Figure 2.

h Data from their Figure 3a, "Event 2". Quality factor reported by Ferrazzini et al. [1990].

D $R$ Rata from their Figure 9b. Quality factor reported by J. Tary, Personal Communication $27,2018,6: 13 \mathrm{pm}$ T 


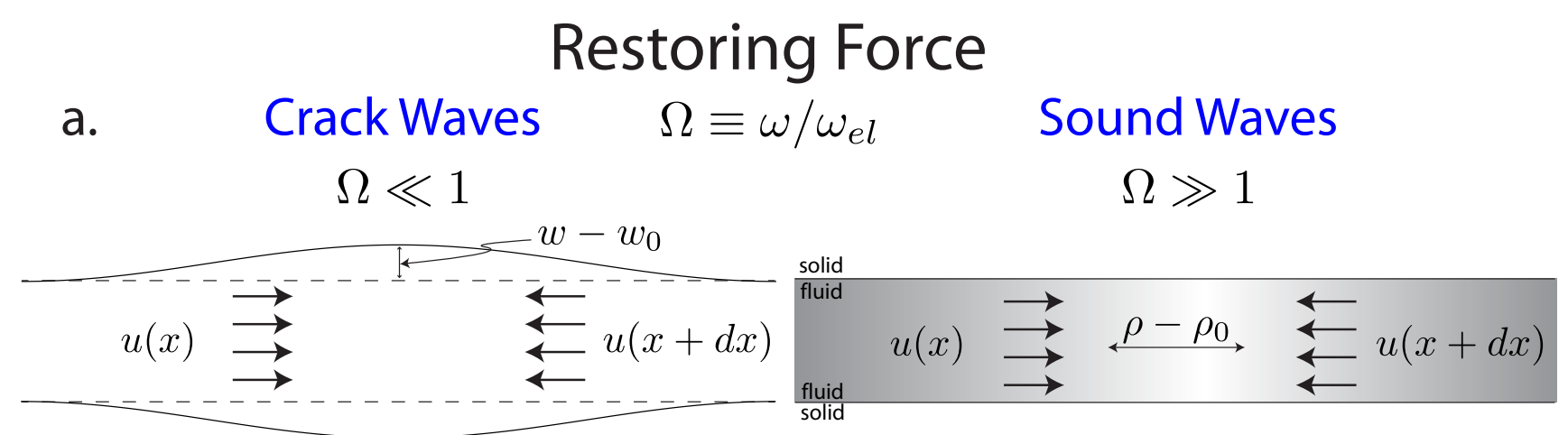

\section{Viscous Damping}

b. Boundary Layer $\quad \zeta \equiv \alpha / \omega \quad$ Fully Developed

$\zeta \ll 1$

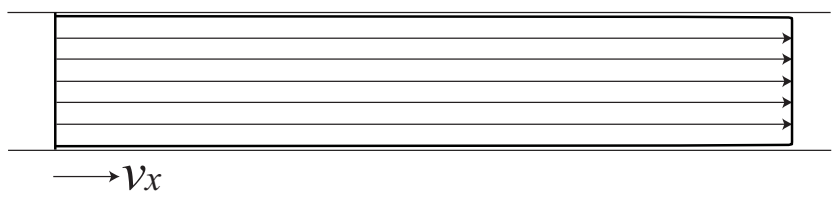

$\zeta \gg 1$

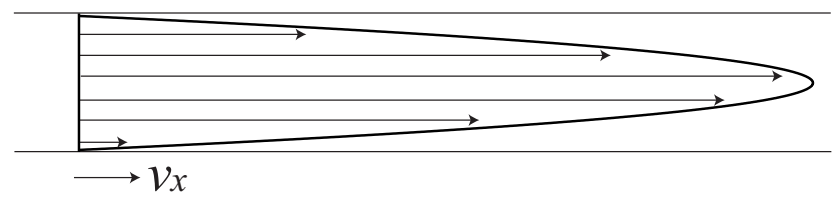

Figure 1. (a.) Two limits of the compressibility ratio $\Omega$ for an inviscid fluid. In the sound wave limit $(\Omega \gg 1)$, the walls are effectively rigid and a convergent velocity field compresses the fluid and increases its density. In the crack wave limit $(\Omega \ll 1)$, the fluid is effectively incompressible and a convergent velocity field pushes the conduit walls apart. Shading indicates the fluid density. (b.) Velocity profiles in the fluid are drawn for a rigid-walled conduit. The left figure shows plug flow with boundary layers. The right plot shows the fully developed, parabolic flow profile.

Table 2. Properties of Solids

\begin{tabular}{llll}
\hline Material & Poisson's Ratio, $\nu_{s}$ & P-wave Speed, $(\mathrm{m} / \mathrm{s})$ & Density, $\rho_{0}\left(\mathrm{~kg} / \mathrm{m}^{3}\right)$ \\
\hline Rock & 0.25 & 5000 & 2700 \\
Ice & 0.35 & 3600 & 920 \\
\hline
\end{tabular}


Table 3. Properties of Fluids

\begin{tabular}{llll}
\hline Material & Viscosity, $\mu(\mathrm{Pa} \cdot \mathrm{s})$ & Sound speed, $c_{0}(\mathrm{~m} / \mathrm{s})$ & Density, $\rho_{0}\left(\mathrm{~kg} / \mathrm{m}^{3}\right)$ \\
\hline Basalt Melt & $10^{2}$ & 1000 & 2500 \\
Andesite Melt & $10^{5}$ & 1000 & 2500 \\
Water & $10^{-3}$ & 1400 & 1000 \\
\hline
\end{tabular}

Table 4. Characteristic Parameters

\begin{tabular}{|c|c|c|c|}
\hline System & $\begin{array}{l}\text { Fracture Half-Width } \\
\left(w_{0}\right)\end{array}$ & Damping Rate $(\alpha)$ & $\begin{array}{l}\text { Elastic Coupling Fre- } \\
\text { quency }\left(\omega_{e l}\right)\end{array}$ \\
\hline $\begin{array}{l}\text { Basalt Melt in Crustal } \\
\text { Rock }\end{array}$ & $1 \mathrm{~m}$ & $0.04 \mathrm{~s}^{-1}$ & $83 \mathrm{~s}^{-1}$ \\
\hline $\begin{array}{l}\text { Andesite Melt in } \\
\text { Crustal Rock }\end{array}$ & $1 \mathrm{~m}$ & $40 \mathrm{~s}^{-1}$ & $83 \mathrm{~s}^{-1}$ \\
\hline Water in Ice & $1 \mathrm{~mm}$ & $1.0 \mathrm{~s}^{-1}$ & $6.4 \times 10^{5} \mathrm{~s}^{-1}$ \\
\hline Water in Rock & $1 \mathrm{~mm}$ & $1.8 \mathrm{~s}^{-1}$ & $9.2 \times 10^{4} \mathrm{~s}^{-1}$ \\
\hline
\end{tabular}

Table 5. Table of symbols

\begin{tabular}{ll}
\hline Symbol & Name \\
\hline$a$ & Pressure-fluid velocity transfer function \\
$b$ & Wall velocity-fluid velocity transfer function \\
$c$ & Phase velocity \\
$c_{0}$ & Fluid sound wave phase velocity \\
$c_{w}$ & Crack wave phase velocity \\
$D$ & Dispersion relation \\
$f$ & Frequency \\
$G^{*}$ & Elastic plane strain modulus \\
$k$ & Wavenumber \\
$k_{c}$ & Cutoff wavenumber \\
$K$ & Nondimensional wavenumber \\
$K_{f}$ & Fluid bulk modulus \\
$L$ & Fracture length \\
$M_{0}$ & Seismic moment \\
$p_{0}$ & Unperturbed fluid pressure \\
$Q$ & Quality factor \\
$t$ & Time \\
$T$ & Damping function \\
$u$ & Width-averaged velocity \\
$u_{x}$ & Wall displacement, $x$-direction \\
$u_{y}$ & Wall displacement, $y$-direction \\
$v_{x}$ & Fluid velocity, $x$-direction \\
$v_{y}$ & Fluid velocity, $y$-direction \\
$w$ & Fracture half-width \\
$x$ & Unperturbed fracture half-width \\
$y$ & Along-conduit coordinate \\
$z$ & Cross-conduit coordinate \\
\hline & Out-of-plane coordinate \\
\hline &
\end{tabular}


Table 6. Table of symbols (continued)

\begin{tabular}{ll}
\hline Symbol & Name \\
\hline$\alpha$ & Viscous damping rate \\
$\gamma$ & Velocity ratio \\
$\Delta(\cdot)$ & Difference from unperturbed state \\
$\epsilon$ & Conduit aspect ratio \\
$\lambda$ & Wavelength \\
$\lambda_{e l}$ & Elastic coupling length \\
$\Lambda$ & Restoring force ratio \\
$\mu$ & Dynamic viscosity \\
$\nu$ & Kinematic viscosity \\
$\nu_{s}$ & Poisson's ratio \\
$\rho$ & Fluid density \\
$\rho_{0}$ & Unperturbed fluid density \\
$\omega$ & Angular frequency \\
$\omega_{e l}$ & Elastic coupling frequency \\
$\Omega$ & Nondimensional frequency, relative to $\omega_{e l}$ \\
$\sigma_{i j}$ & Solid stress tensor \\
$\tau$ & Wall shear stress \\
$\xi$ & Complex damping parameter \\
$\zeta$ & Damping ratio \\
$\zeta_{*}$ & Damping ratio scale \\
$(\cdot)^{\prime}$ & Nondimensional quantity \\
$(\cdot)_{B L}$ & Characteristic amplitude, boundary layer limit \\
$(\cdot)_{F D}$ & Characteristic amplitude, fully developed flow \\
$(\cdot)_{R}$ & limit \\
$(\cdot)_{I}$ & Real part \\
$(\cdot)_{n}$ & Imaginary part \\
& Pertaining to n ${ }^{\text {th }}$ harmonic \\
\hline & \\
& \\
\hline
\end{tabular}



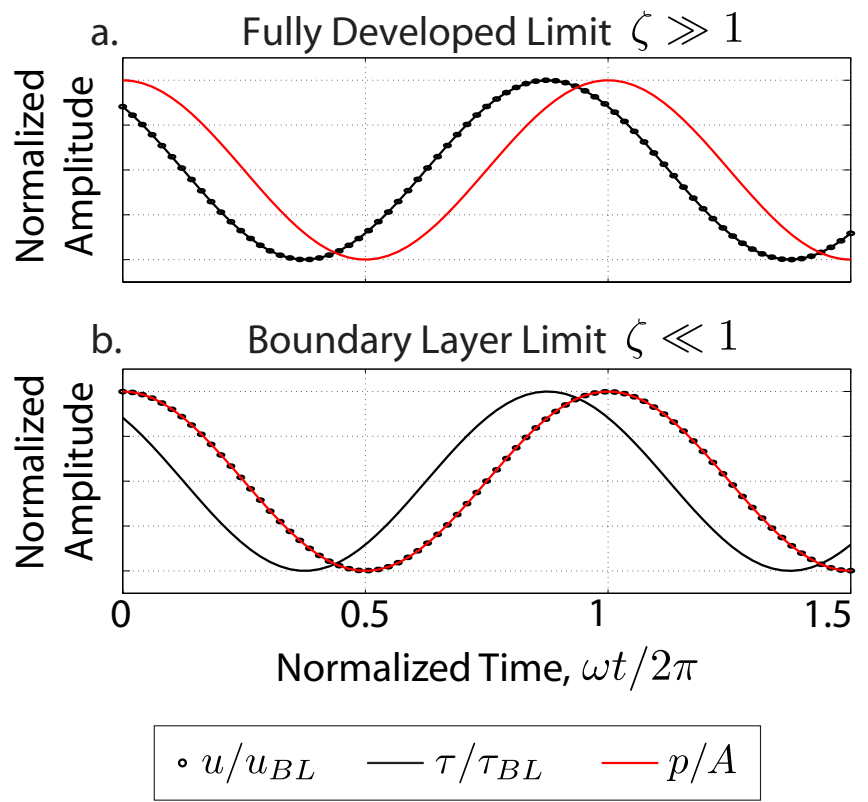

Figure 2. The phase relationships between pressure, velocity, and shear stress for a time harmonic wave having angular frequency $\omega$ and pressure amplitude $A$. In the fully developed flow limit $(\zeta \gg 1)$, velocity and shear stress lead the pressure perturbation. In the boundary layer limit $(\zeta \ll 1)$, pressure and velocity are in phase. The fully developed and boundary layer characteristic velocities and shear stresses (42)-(45) are used to normalize wave amplitudes. The motions arise from the dispersion relation (57) with complex wavenumber. The phase relationships to not depend on $\Omega$. 

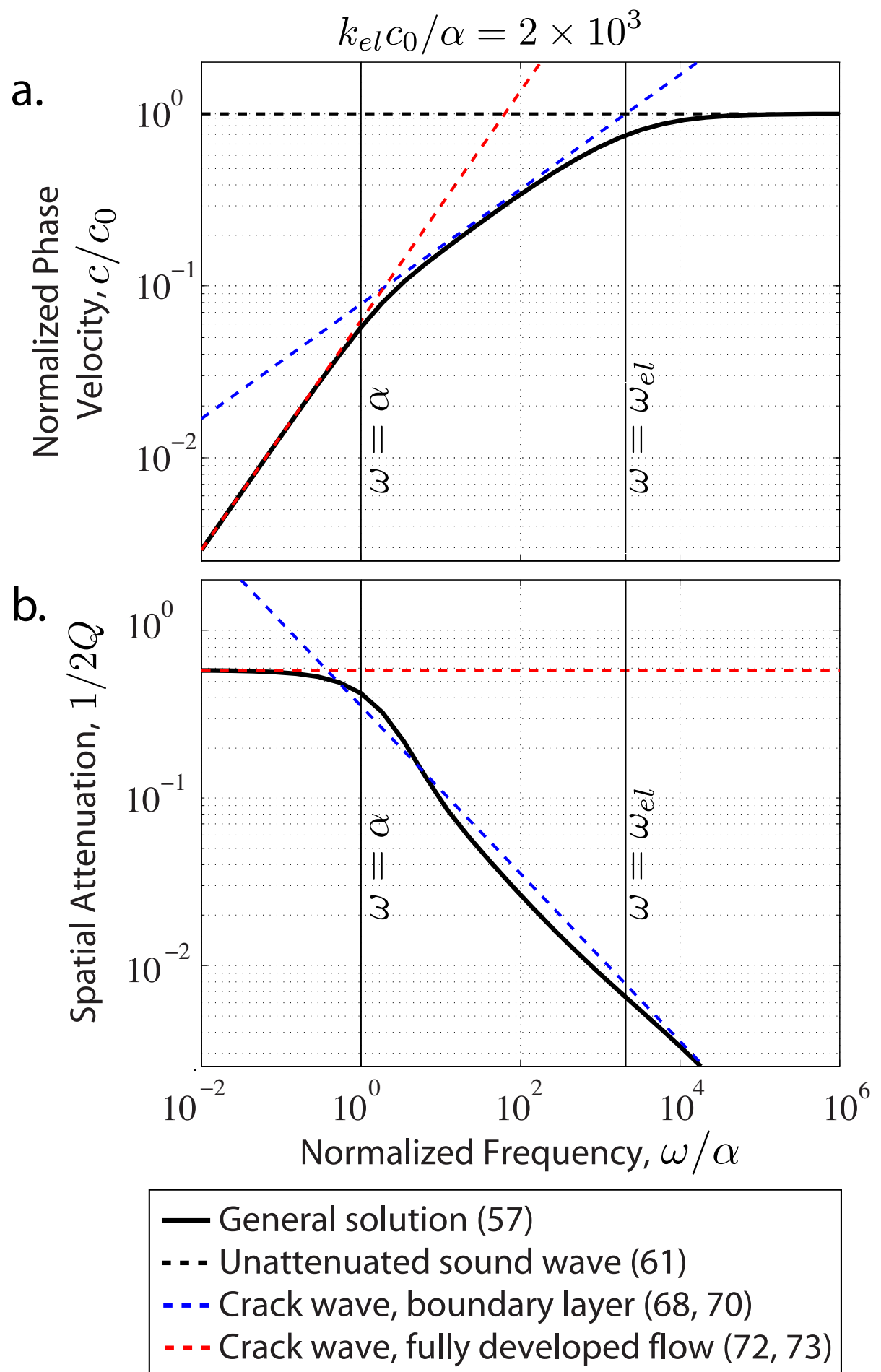

Figure 3. Phase velocity (a.) and spatial attenuation (b.) plotted against frequency. The figure is drawn for a $1 \mathrm{~m}$ wide basaltic melt-filled conduit in rock (Tables 2 and 3). Several asymptotic limits are plotted. Not shown is the sound wave, boundary layer limit (63) because spatial attenuation in this limit closely follows the crack wave, boundary layer limit (70). 


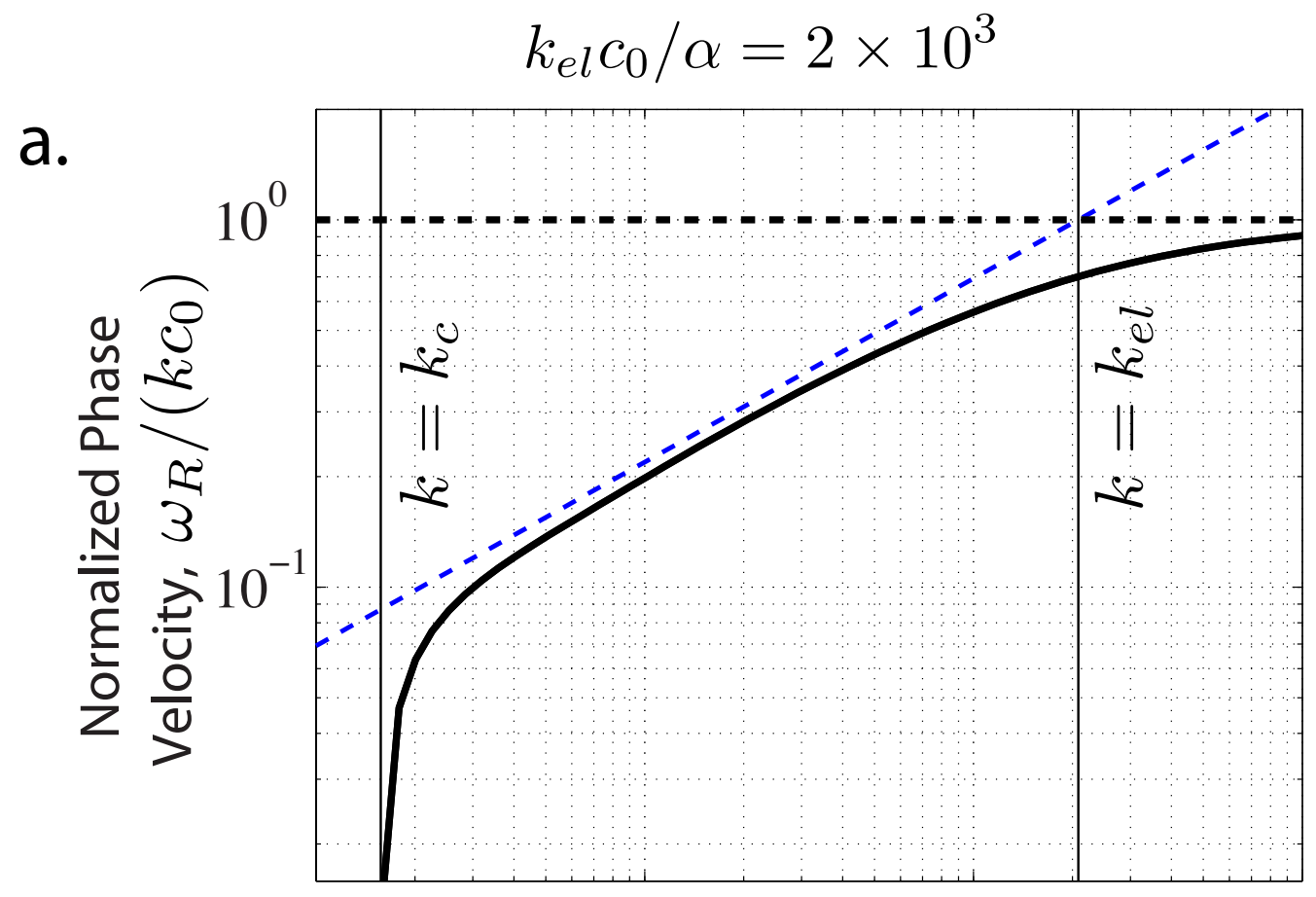

b.

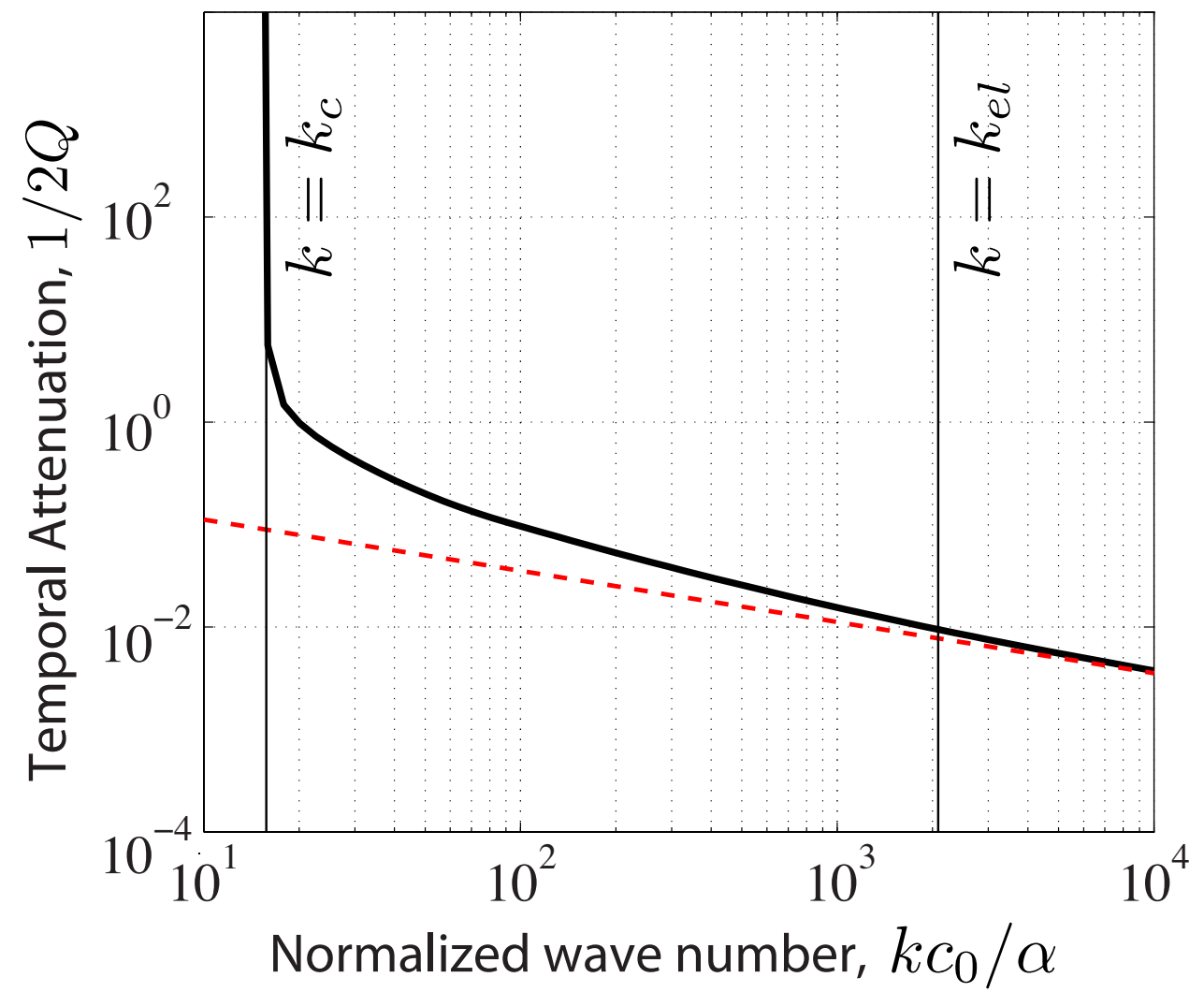

-General solution of (76)

- - -Crack wave limit (78)

- - -Sound wave limit (77)

- - - Boundary layer limit (81)

Figure 4. Phase velocity (a) and temporal attenuation (b) of waves with real wavenumber May 27, 2018, 6:13pm
plotted a F T ainst wavenumber. The vertical lines show the cutoff wavenumber (left, Equation $855^{\text {) }}$ ) and $k=k_{e l}$ (right). The figure is drawn for a $1 \mathrm{~m}$ wide basaltic melt-filled conduit in rock (Tables 


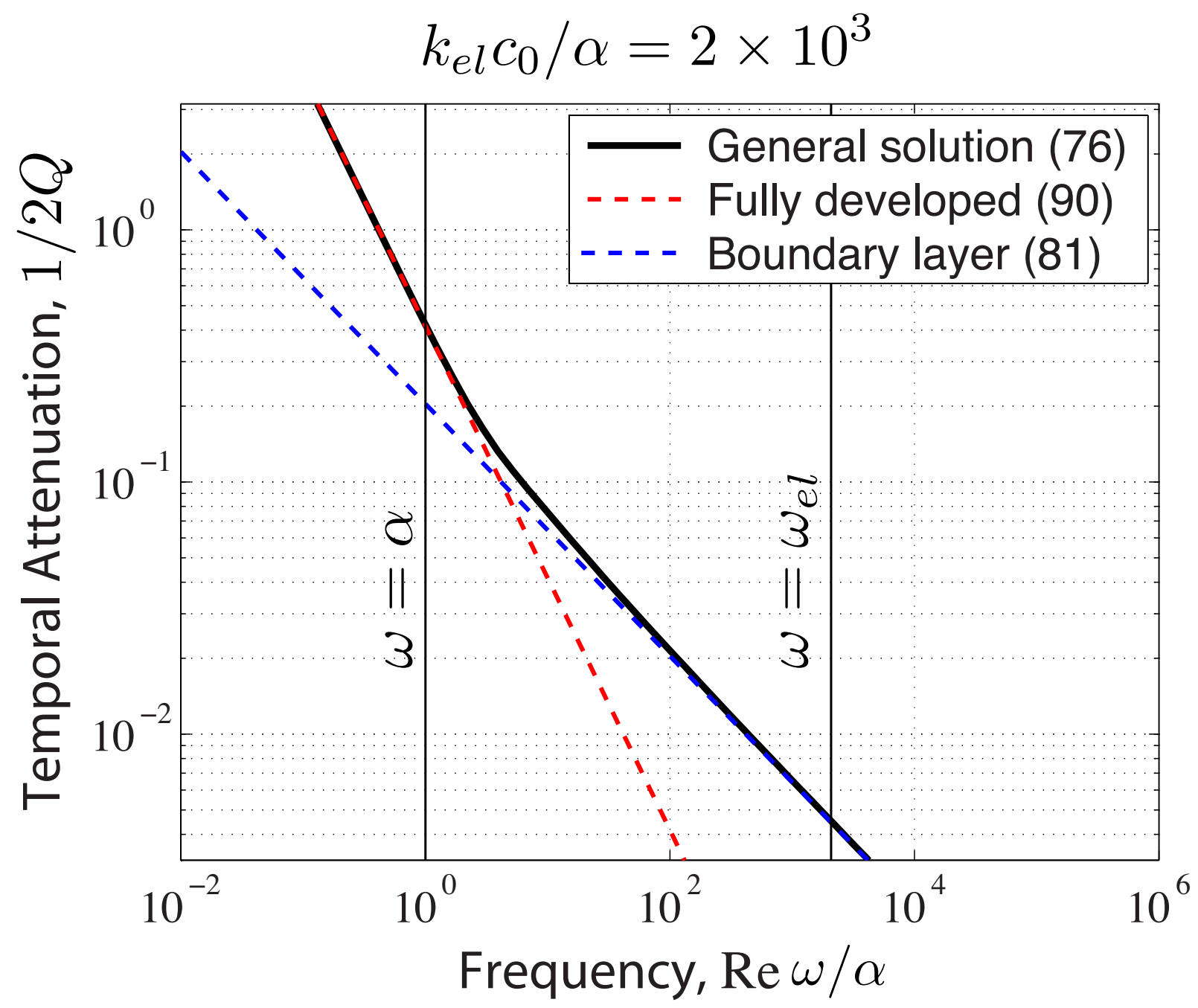

Figure 5. Temporal attenuation plotted against frequency of oscillation $\omega_{R}$ for waves with real-valued wavenumber $k$. Attenuation is calculated as a function of a real wavenumber using (76). The boundary layer limit spatial attenuation is the same as the boundary layer limit temporal attenuation (blue dashed line, Equation 63). The fully developed flow limit has temporal attenuation shown with a red dashed line (90). The figure is drawn for a $1 \mathrm{~m}$ wide basaltic melt-filled conduit in rock (Tables 2 and 3). 
a.

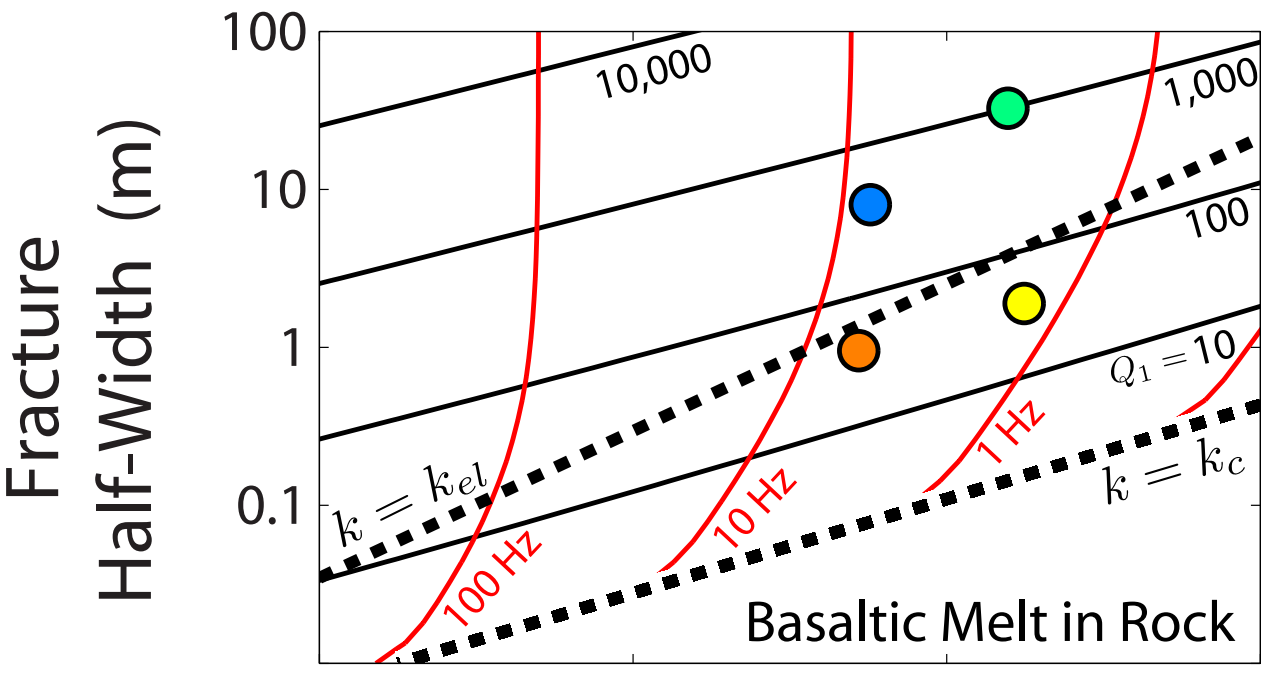

b.

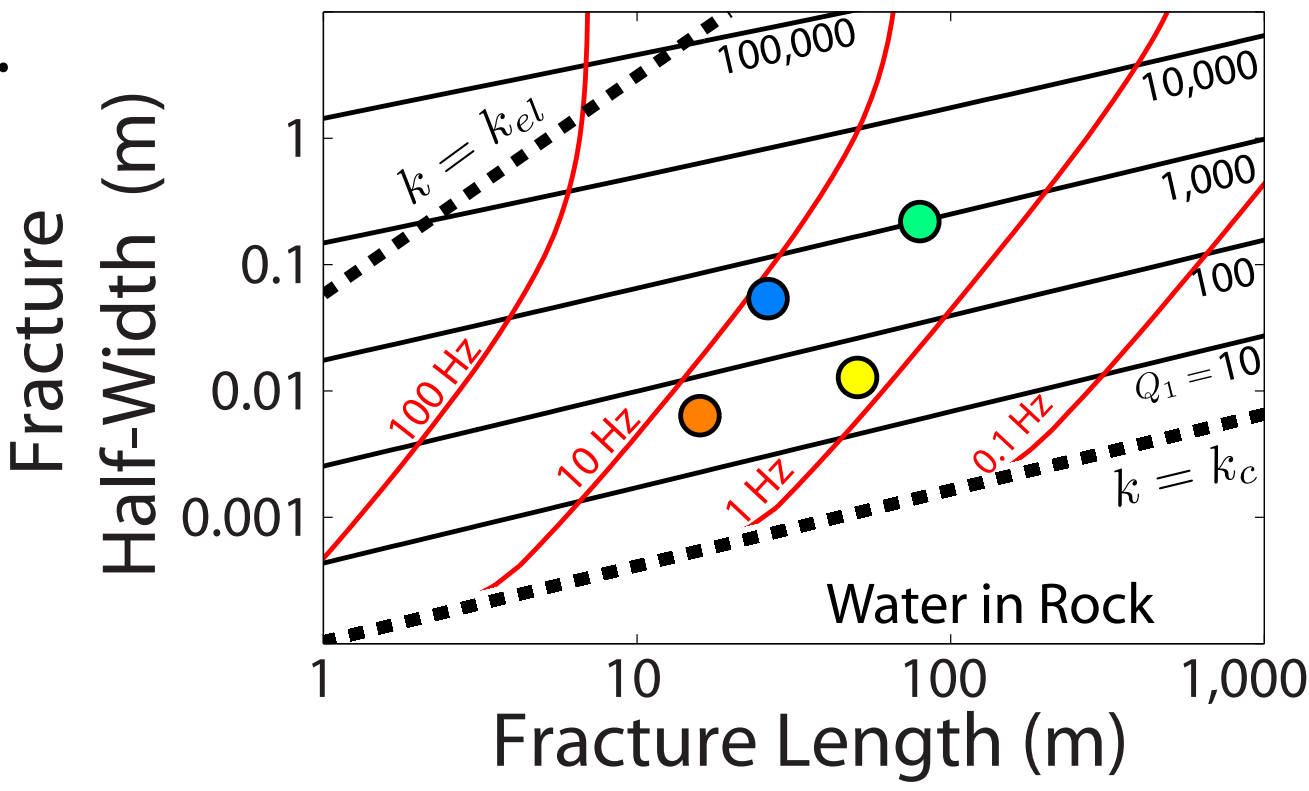

\section{OGaleras OKilauea \\ OKusatsu-Shirane ORedoubt}

Figure 6. Plot to infer fracture half-width and length of magmatic hydraulic fractures from observed characteristic seismic frequency $f_{1}$ and quality factor $Q_{1}$. Observations and inferred parameters are listed in Table 1. We carry out calculations for two different fluids: a. basalt melt and b. water. 


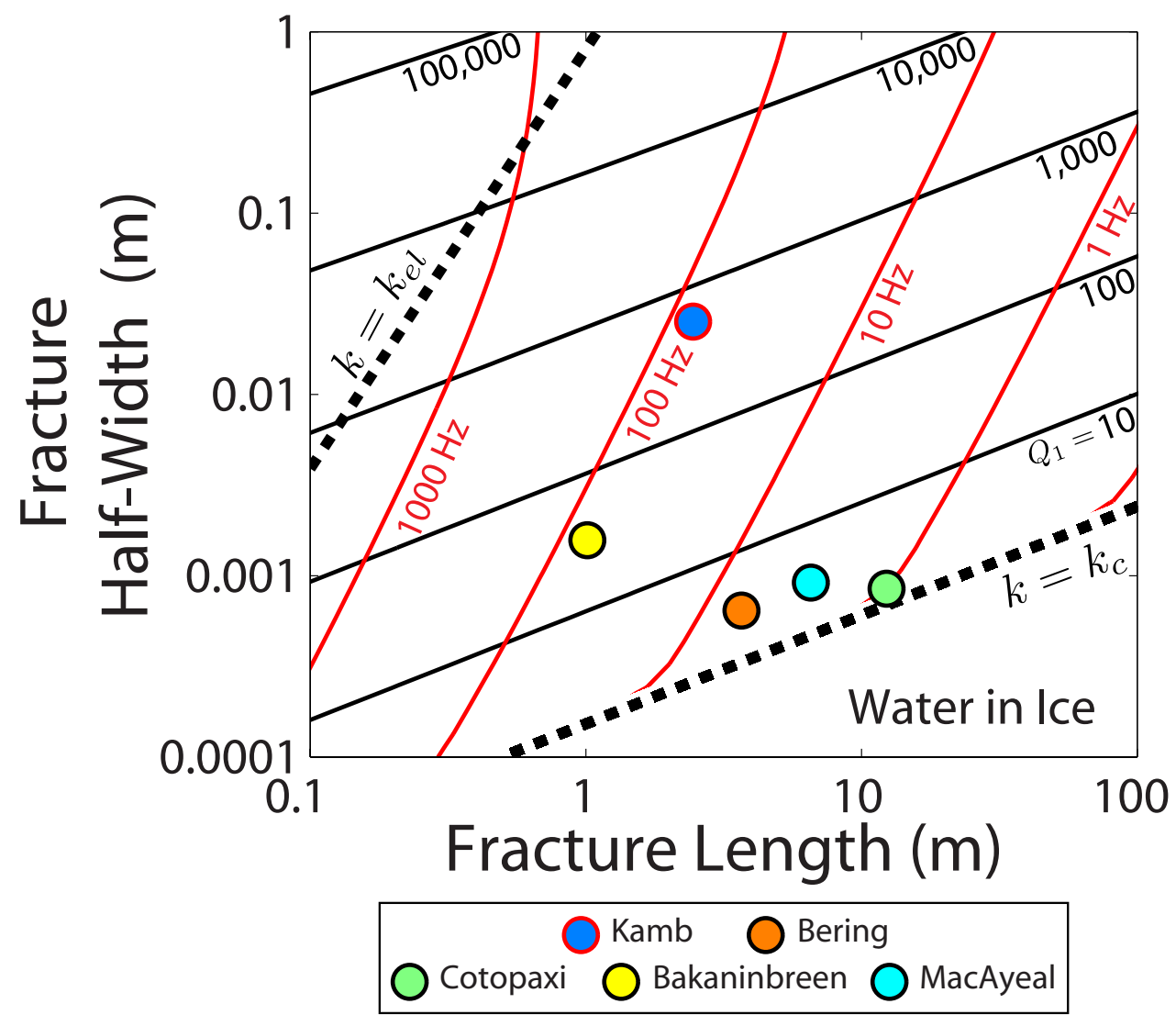

Figure 7. Plot to infer fracture half-width and length of glacial hydraulic fractures from observed characteristic seismic frequency $f_{1}$ and quality factor $Q$. Observations and inferred parameters are listed in Table 1. Data points with red outlines have evenly spaced spectral peaks and therefore may not correspond to hydraulic fracture resonance. 


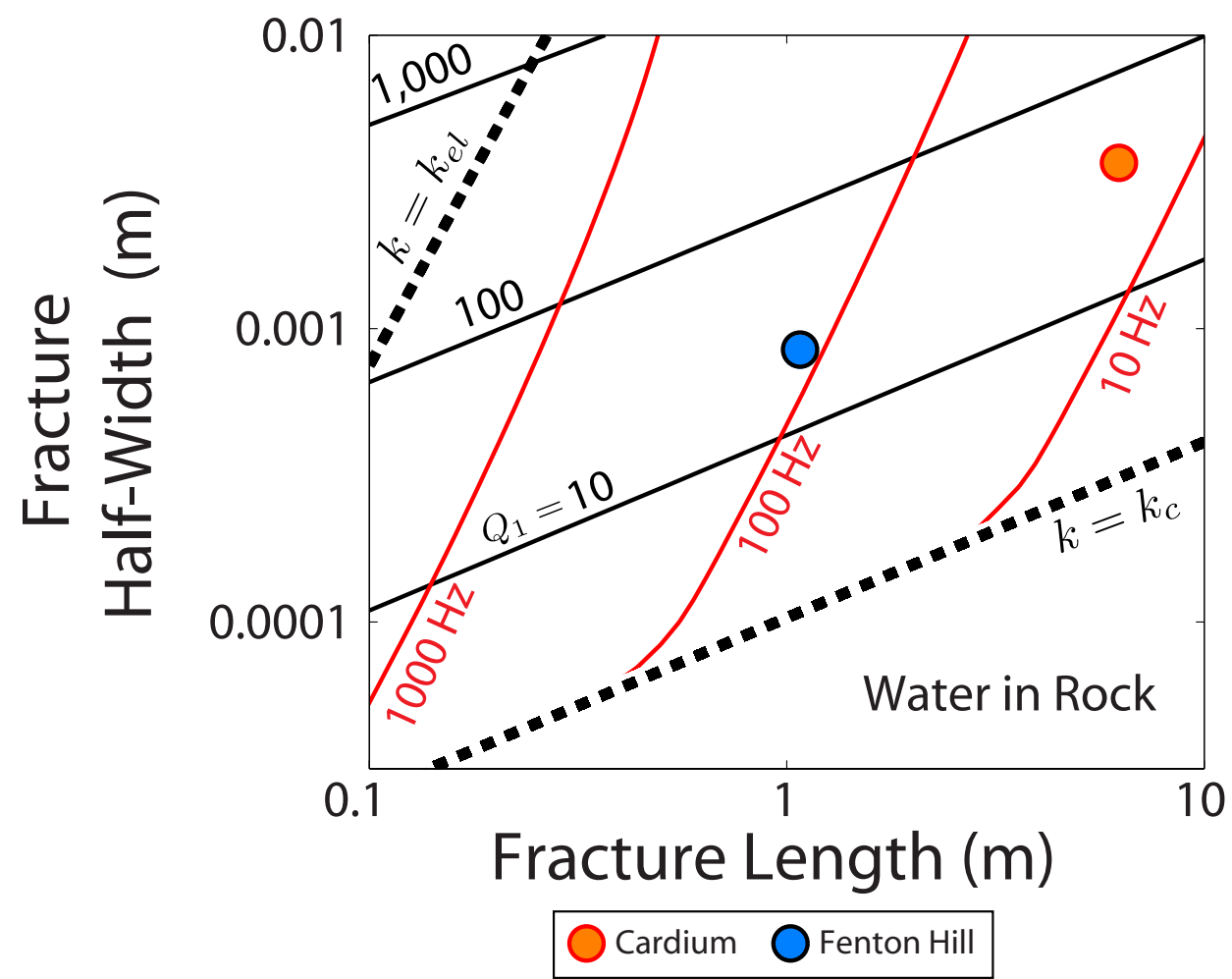

Figure 8. Plot to infer fracture half-width and length in several reservoirs from observed characteristic seismic frequency $f_{1}$ and quality factor $Q$, assuming water-filled fractures in rock. Observations and inferred parameters are listed in Table 1. Data points with red outlines have evenly spaced spectral peaks and therefore may not correspond to hydraulic fracture resonance. 

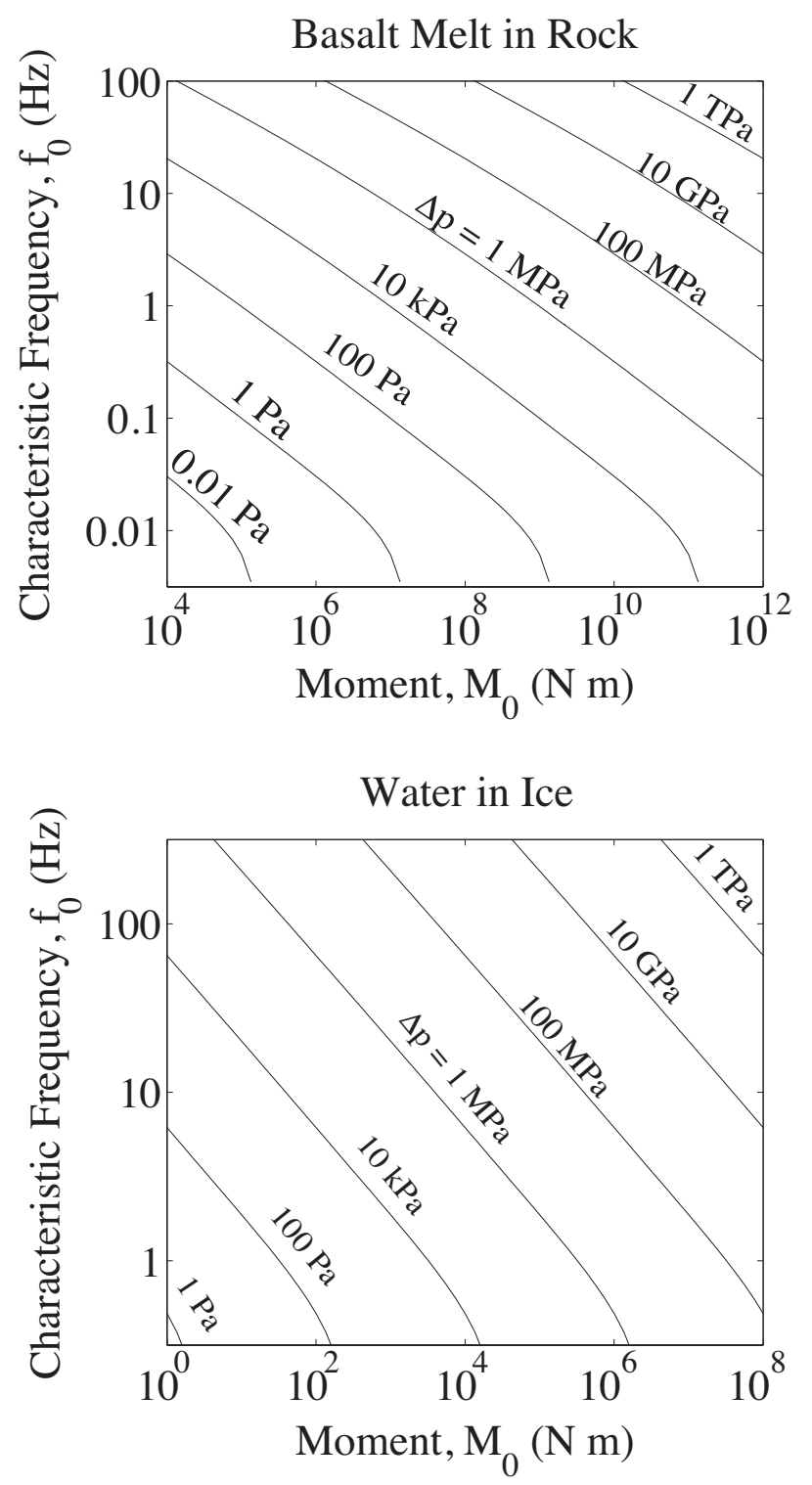

Figure 9. Plot to infer fracture pressure changes $\Delta p$ from seismically observed characteristic frequency $f_{1}$ and moment $M_{0}$. Curves of constant pressure perturbation are drawn from (97). 